International Journal of Bifurcation and Chaos, Vol. 12, No. 7 (2002) 1579-1597

(c) World Scientific Publishing Company

\title{
ADAPTIVE SYNCHRONIZATION FOR RÖSSLER AND CHUA'S CIRCUIT SYSTEMS
}

\author{
A. S. HEGAZI*, H. N. AGIZA ${ }^{\dagger}$ and M. M. EL-DESSOKY ${ }^{\ddagger}$ \\ Mathematics Department, Faculty of Science, \\ Mansoura University, Mansoura, 35516, Egypt \\ *hegazi@mum.mans.eun.eg \\ †agizah@mum.mans.eun.eg \\ $\ddagger$ dessokym@mum.mans.eun.eg
}

Received February 23, 2001; Revised August 14, 2001

\begin{abstract}
This study addresses the adaptive synchronization of Rössler and Chua circuit systems with unknown parameters. By using Lyapunov stability theory the adaptive synchronization law with a single-state variable feedback is derived, such that the trajectory of the two systems are globally stabilized to an equilibrium point of the uncontrolled system (globally stable means that the method of the solution is restricted in area of phase space i.e. globally in a subset of a phase space with bounded zero volume). We use the Lyapunov direct method to study the asymptotic stability of the solutions of error system. Numerical simulations are given to explain the effectiveness of the proposed control scheme.
\end{abstract}

Keywords: Chaotic systems; adaptive synchronization; Rössler system; Chua's circuit system; error system; Lyapunov direct method.

\section{Introduction}

We define in general terms the concept of synchronization of the dynamical variables in two dynamical systems which can exhibit either chaotic or nonchaotic behavior.

Synchronization can occur within a subset of dynamical variables instead of all of them. In order to make our technique have some partical use we approach synchronization stresses with the use of controlling forces that are given in terms of observable. We apply synchronization to two different nonlinear systems separately, first the Rössler system and second, the Chua circuit which can exhibit chaos.

In all cases, the controlling forces that we use are expressed in terms of the difference of the instantanceous value of a dynamical variable in one system, called the driving system, and the corresponding variable in the other system, called the controlled system. The parameters of both systems are assumed to be identical, but unknown. We present some methods that are successful if a Lyapunov function can be shown to exist. If the Lyapunov function exists globally, then the synchronization occurs globally. The specific Lyapunov functions presented for the specific systems, the Rössler and the Chua circuit systems, are decreasing functions of time only in restricted regions of phase space, i.e. "globally" in a subset of phase space where the volume is zero.

Chaos has been thoroughly studied over the past two decades [Chen \& Dong, 1993; Chen, 1997]. A chaotic system is a nonlinear deterministic system that displays complex, noise-like and unpredictable behavior. The sensitive dependence upon initial conditions and on the variations of the system's parameter are prominent characteristics of chaotic behavior. Researchers have investigated the "chaos 
control" and "chaos synchronization" problems in many physical chaotic systems [Pecora \& Carroll, 1990; Carroll \& Pecora, 1991; Chen \& Dong, 1993; Kapitaniak, 1995; Xie \& Chen, 1996; Chen, 1997].

The idea of synchronizing two identical systems that start from different initial conditions was introduced by Pecora and Carroll [1990]. It investigates the linking of the trajectory of one system to the other system with the same values parameter (solution), such that they remain together in each step through the transmission of a signal.

Synchronization in chaotic dynamical systems has received considerable attention in nonlinear sciences in the last decade [Pecora \& Carroll, 1991; Pyragas, 1992; Cuomo \& Oppenheim, 1993; Kocarev \& Parlitz, 1995; Femat \& Solis-Perales, 1999].

The concept of chaos synchronization involves making two chaotic systems oscillate in a synchronized manner. It holds promise for creating secure communication systems [Wu et al., 1996; Zeng \& Singh, 1996].

One of the important issues in the study of synchronization of dynamical systems of unknown system parameters is the adaptive synchronization of chaotic dynamical systems. Some papers [Liao \& Lin, 1999; Hegazi et al., 2001] have derived an adaptive scheme for synchronization in the control of two chaotic systems with mismatched parameters. An adaptative mechanism can compensate for the effects of those parametric uncertainties. The control scheme was then successfully applied to the Lorenz system [Liao \& Lin, 1999] and Nuclear Spin Generator system [Hegazi et al., 2001]. In this work we apply the control scheme to Rössler and Chua circuit systems.

Consider the following system

$$
\dot{x}=f(x), \quad x \in R^{n}, \quad f \in c^{1}[R, R]
$$

Instead of state variables $x$, we consider $m$ scalar functions $S_{1}(x), \ldots, S_{m}(x)$ versus state variables $x \in R^{n}$. These $m$ functions are observable and are also called synchronizable variables here. Since what should be of concern in a system are the variables that are observable, the synchronization is thus naturally defined as follows [Yang, 1999, 2000].

Definition 1. Two systems

$$
\begin{aligned}
& \dot{x}=f(x), \quad x \in R^{n}, \quad f \in c^{1}[R, R] \\
& \dot{y}=G(y), \quad y \in R^{n}, \quad G \in c^{1}[R, R]
\end{aligned}
$$

are said to be synchronizable with respect to observable variables $S_{1}(x), S_{2}(x), \ldots, S_{m}(x)$ by a coupling $\left(c_{1}(x, y), c_{2}(x, y)\right)$, if

$$
\lim _{t \rightarrow \infty}\left\|S_{i}(x(t))-S_{i}(y(t))\right\|=0, \quad i=1,2, \ldots, m .
$$

Here the coupling takes the form of

$$
\begin{aligned}
& \dot{x}=f\left(x, c_{1}(x, y)\right) \\
& \dot{y}=G\left(y, c_{2}(x, y)\right)
\end{aligned}
$$

More generally, the synchronization can be defined between two (or more) subsystems of a large system

Definition 2. Consider the following system

$$
\begin{aligned}
& \dot{x}=f(x, y) \\
& \dot{y}=G(x, y), \quad(x, y) \in R^{n} \times R^{n}
\end{aligned}
$$

The state variables $x$ and $y$ are said to be synchronizable with respect to observable variables $S_{1}, S_{2}, \ldots, S_{m}$ if the relation

$$
\lim _{t \rightarrow \infty}\left\|S_{i}(x(t))-S_{i}(y(t))\right\|=0, \quad i=1,2, \ldots, m
$$

holds.

In view of the above definitions, an important practical question arises: what variable is observable and meaningful versus state variables? This apparently depends on what system is used.

Taking $S_{i}(x)$ to be $x_{i}$, and $S_{i}(y)$ to be $y_{i}, i=$ $1,2, \ldots, n$, we then return to the popular concept of synchronization in the current literature. Clearly, the phase locking and the frequency locking are special cases of the above definitions.

The concept of synchronization, in its broadest meaning, should be defined as follows.

Definition 3. Consider system

$$
\begin{aligned}
& \dot{x}=f(x, y) \\
& \dot{y}=G(x, y), \quad(x, y) \in R^{n} \times R^{n}
\end{aligned}
$$

Let $\quad Q_{1}(x), Q_{2}(x), \ldots, Q_{h}(x) \quad$ and $\quad S_{1}(y)$, $S_{2}(y), \ldots, S_{h}(y)$ be observable variables of systems (5) and (6), respectively, which possess the same physical meaning. The systems (5) and 
(6) are said to be synchronizable with respect to $\left(Q_{1}, Q_{2}, \ldots, Q_{h}\right)$ and $\left(S_{1}, S_{2}, \ldots, S_{h}\right)$, if

$$
\lim _{t \rightarrow \infty}\left\|Q_{i}(x(t))-S_{i}(y(t))\right\|=0, \quad i=1,2, \ldots, h .
$$

It is easy to see that Definition 3 includes the known notion of generalized synchronization as its special case [Kocarev \& Pralitz, 1996; Pralitz et al., 1997].

Proposition 1. Let $S_{1}, S_{2}, \ldots, S_{m}$ be observable variables for systems (3) and (4). Let $V\left(x_{1}, x_{2}, \ldots, x_{m}\right)$ be a positive definite Lyapunov function with $V(0)=0$. Suppose that

$$
\begin{gathered}
\frac{d}{d t} V\left(S_{1}(x(t))-S_{1}(y(t)), S_{2}(x(t))-S_{2}(y(t)), \ldots,\right. \\
\left.S_{m}(x(t))-S_{m}(y(t))\right) \leq 0
\end{gathered}
$$

where the equality holds only if $S_{i}(x(t))=$ $S_{i}(y(t)), i=1,2, \ldots, m$. Then systems (3) and (4) are synchronizable with respect to $S_{1}, S_{2}, \ldots, S_{m}$.

Proposition 2. Let $\left(Q_{1}, Q_{2}, \ldots, Q_{h}\right)$ and $\left(S_{1}, S_{2}, \ldots, S_{h}\right)$ be two groups of observable variables for systems (5) and (6), respectively. Suppose that there exists a positive definite Lyapunov function $V\left(x_{1}, x_{2}, \ldots, x_{h}\right)$ such that

$$
\begin{gathered}
\frac{d}{d t} V\left(Q_{1}(x(t))-S_{1}(y(t)), Q_{2}(x(t))-S_{2}(y(t)), \ldots,\right. \\
\left.Q_{h}(x(t))-S_{h}(y(t))\right) \leq 0
\end{gathered}
$$

and the equality holds only if $\left(Q_{i}(x(t))-S_{i}(y(t))\right)=$ $0, i=1,2, \ldots, h$. Then systems (5) and $(6)$ are synchronizable with respect to $\left(Q_{1}, Q_{2}, \ldots, Q_{h}\right)$ and $\left(S_{1}, S_{2}, \ldots, S_{h}\right)$.

Proposition 3. Let $\left(Q_{1}, Q_{2}, \ldots, Q_{h}\right)$ and $\left(S_{1}\right.$, $\left.S_{2}, \ldots, S_{k}\right)$ be two groups of observable variables for systems (5) and (6), respectively. Suppose that there is a transformation $F: R^{h} \rightarrow R^{k}$ and positive definite Lyapunov function $V\left(x_{1}, x_{2}, \ldots, x_{k}\right)$ such that

$$
\begin{array}{r}
\frac{d}{d t} V\left(T _ { 1 } \left(Q(x(t))-S_{1}(y(t)), \ldots,\right.\right. \\
T_{h}\left(Q(x(t))-S_{k}(y(t))\right) \leq 0
\end{array}
$$

and the equality holds only if $T_{i}(Q(x(t))-$ $\left.S_{i}(y(t))\right)=0, i=1,2, \ldots, k$. Then systems (5) and $(6)$ are generalized synchronizable with respect to $\left(Q_{1}, Q_{2}, \ldots, Q_{h}\right)$ and $\left(S_{1}, S_{2}, \ldots, S_{k}\right)$.

Proposition 4. Suppose that the orbit pair $x\left(t, x_{0}, y_{0}\right)$ and $y\left(x_{0}, y_{0}\right)$ in systems (1) and (2) are synchronizable with respect to observable variables $S_{1}, S_{2}, \ldots, S_{m}$, then the omega limit set $\Omega\left(x_{0}, y_{0}\right)$ lies in the set $M_{S}=\left\{(x, y): S_{i}(x)=S_{i}(y)\right.$, $\left.(x, y) \in R^{n} \times R^{n}, i=1, \ldots, m\right\}$. And on this set $\Omega\left(x_{0}, y_{0}\right)$, the following holds

$$
F(x, y) \cdot \operatorname{grad} S_{i}(x)-G(x, y) \cdot \operatorname{grad} S_{i}(y)=0
$$

where $(x, y) \in \Omega\left(x_{0}, y_{0}\right)$.

Proposition 5. Suppose that the orbit pair $x\left(t, x_{0}, y_{0}\right)$ and $y\left(x_{0}, y_{0}\right)$ in systems (5) and (6) are synchronizable with respect to observable variables $Q_{1}(x), Q_{2}(x), \ldots, Q_{h}(x)$ and $S_{1}(y), S_{2}(y), \ldots, S_{h}(y)$. Then the omega limit set $\Omega\left(x_{0}, y_{0}\right)$ lies in the set $M_{Q S}=\left\{(x, y): Q_{i}(x)=\right.$ $\left.S_{i}(y),(x, y) \in R^{m} \times R^{n}, i=1, \ldots, h\right\}$. And on this set $\Omega\left(x_{0}, y_{0}\right)$, the following holds

$$
F(x, y) \cdot \operatorname{grad} Q_{i}(x)-G(x, y) \cdot \operatorname{grad} S_{i}(y)=0
$$

where $(x, y) \in \Omega\left(x_{0}, y_{0}\right)$.

The above criteria are of more theoretical interest. The aim of this paper is to have a practical significance, so we control and synchronize the chaotic dynamical system Rössler and Chua circuit by using adaptive synchronization. The Lyapunov direct method is used to prove the asymptotic behavior of the solutions for the controlled system. We apply this technique for Rössler and Chua circuit systems of differential equations and drag the trajectories of the error system to the zero solution.

\section{Adaptive Synchronization of Rössler System}

Let us consider the Rössler dynamical system [Xie \& Chen, 1996] which is given by the autonomous differential equations:

$$
\begin{aligned}
& \dot{x}=-y-z \\
& \dot{y}=x+a y \\
& \dot{z}=b+(x-c) z
\end{aligned}
$$

where $a, b$ and $c$ are positive constants.

The system of differential equations (7) has two equilibrium points: $\left(x_{1}, y_{1}, z_{1}\right)=\left(a \sigma_{-},-\sigma_{-}, \sigma_{-}\right)$ and $\left(x_{2}, y_{2}, z_{2}\right)=\left(a \sigma_{+},-\sigma_{+}, \sigma_{+}\right)$where $\sigma_{+}=c+$ $\sqrt{c^{2}-4 a b} / 2 a$ and $\sigma_{-}=c-\sqrt{c^{2}-4 a b} / 2 a$. 
For $c^{2}>4 a b$, we get $\sigma_{+}>0$ and $\sigma_{-}>0$, hence $x_{i}>0, y_{i}<0$ and $z_{i}>0, i=1,2$. The equilibrium point $\left(x_{1}, y_{1}, z_{1}\right)$ is unstable solution for the system (7).

In order to observe the synchronization behavior in Rössler systems [Wu et al., 1996; Liao \& Lin, 1999], we have two Rössler systems where the drive system with three state variables denoted by the subscript 1 drives the response system having identical equations denoted by the subscript 2 . However, the initial condition of the drive system is different from that of the response system; therefore two Rössler systems are described, respectively, by the following equations:

$$
\begin{aligned}
& \dot{x}_{1}=-y_{1}-z_{1} \\
& \dot{y}_{1}=x_{1}+a y_{1} \\
& \dot{z}_{1}=b+\left(x_{1}-c\right) z_{1}
\end{aligned}
$$

and

$$
\begin{aligned}
& \dot{x}_{2}=-y_{2}-z_{2}+u_{1} \\
& \dot{y}_{2}=x_{2}+a y_{2}+u_{2} \\
& \dot{z}_{2}=b+\left(x_{2}-c\right) z_{2}+u_{3}
\end{aligned}
$$

We have introduced three control inputs, $u_{1}$, $u_{2}$ and $u_{3}$ in Eq. (9). $u_{1}, u_{2}$ and $u_{3}$ are to be determined for the purpose of synchronizing the two identical Rössler systems with the same but unknown parameters $a, b$ and $c$ in spite of the differences in initial conditions.

Remark 1. The Rössler system has a bounded, zero volume, globally attracting set [Xie \& Chen, 1996]. Therefore, the state trajectories $x(t), y(t)$ and $z(t)$ are globally bounded for all $t \geq 0$ and continuously differentiable with respect to time. Consequently, there exist three positive constants $s_{1}, s_{2}$ and $s_{3}$ such that $|x(t)| \leq s_{1}<\infty,|y(t)| \leq s_{2}<\infty$ and $|z(t)| \leq s_{3}<\infty$ hold for all $t \geq 0$.

Let us define the state errors between the response system that is to be controlled and the controlling drive system as

$$
e_{x}=x_{2}-x_{1}, \quad e_{y}=y_{2}-y_{1} \quad \text { and } \quad e_{z}=z_{2}-z_{1}
$$

Subtracting Eq. (8) from Eq. (9) and using the notation (10) yields

$$
\begin{aligned}
& \dot{e}_{x}=-e_{y}-e_{z}+u_{1} \\
& \dot{e}_{y}=e_{x}+a e_{y}+u_{2} \\
& \dot{e}_{z}=z_{1} e_{x}+\left(x_{2}-c\right) e_{z}+u_{3}
\end{aligned}
$$

Hence the synchronization problem is now replaced by an equivalent problem of stabilizing the system (11), by using a suitable choice of the control laws $u_{1}, u_{2}$ and $u_{3}$. Let us now discuss the following three cases of control inputs $u_{1}, u_{2}$ and $u_{3}$ :

\subsection{First case}

The state variable $x_{1}$ of the drive system is coupled to the first equation of the response system, and an external control with the state $x_{2}$ as the feedback variable is introduced into the first equation in (11). Therefore, the feedback control law is described as

$$
u_{1}=-\tilde{k}_{1} e_{x}, \quad u_{2}=0 \quad \text { and } \quad u_{3}=0
$$

where $\tilde{k}_{1}$ denotes an estimated feedback gain which is updated according to the following adaptative algorithm

$$
\dot{\tilde{k}}_{1}=\gamma e_{x}^{2}, \quad \tilde{k}_{1}(0)=0
$$

Then the resulting error dynamical system can be expressed as

$$
\begin{aligned}
& \dot{e}_{x}=-\tilde{k}_{1} e_{x}-e_{y}-e_{z} \\
& \dot{e}_{y}=e_{x}+a e_{y} \\
& \dot{e}_{z}=z_{1} e_{x}+\left(x_{2}-c\right) e_{z} \\
& \dot{\tilde{k}}_{1}=\gamma e_{x}^{2}, \quad \tilde{k}_{1}(0)=0
\end{aligned}
$$

We define the Lyapunov function $V$ by

$$
V=\frac{1}{2}\left(e_{x}^{2}+e_{y}^{2}+e_{z}^{2}+\frac{1}{\gamma}\left(\tilde{k}_{1}-k_{1}^{* *}\right)^{2}\right)
$$

In order to control Lyapunov function of the system we define another positive constant $k_{1}^{* *}$ which makes $\dot{V}$ a negative definite $(\dot{V}<0)$. Taking the time derivative of Eq. (15), we get

$$
\begin{aligned}
\dot{V} & =-\left\{k_{1}^{* *} e_{x}^{2}-a e_{y}^{2}+\left(c-x_{2}\right) e_{z}^{2}+\left(1-z_{1}\right) e_{x} e_{z}\right\} \\
& =-\left\{k_{1}^{* *} e_{x}^{2}-a e_{y}^{2}+\left(c-s_{1}\right) e_{z}^{2}+\left(1-s_{3}\right) e_{x} e_{z}\right\} \\
& \leq 0
\end{aligned}
$$

From Remark 1 the response variables are bounded and the derivative $\dot{V} \leq 0$. We deduce the following condition: 
Since

(i) $c-s_{1}>0$

(ii) $1-s_{3}>0$

(iii) $k_{1}^{* *}>0$ and we choose

(iv) $k_{1}^{* *} e_{x}^{2}+\left(c-s_{1}\right) e_{z}^{2}+\left(1-s_{3}\right) e_{x} e_{z}>a e_{y}^{2}$ then $k_{1}^{* *} e_{x}^{2}-a e_{y}^{2}+\left(c-s_{1}\right) e_{z}^{2}+\left(1-s_{3}\right) e_{x} e_{z}>0$

Since $V$ is a positive and decreasing function and $\dot{V}$ is a negative semidefinite, it follows that the equilibrium point $\left(e_{x}=0, e_{y}=0, e_{z}=0, \dot{k}_{1}=k_{1}^{* *}\right)$ of the system (14) is uniformly stable. This implies that the two Rössler systems have been globally asymptotically synchronized under the control law (14) associated with (13).

\subsection{Second case}

The state variable $y_{1}$ of the drive system is coupled to the first equation of the response system and an external control with the state $y_{2}$ as the feedback variable is also introduced into the second equation in (11). Therefore, the feedback control law is described as

$$
u_{1}=0, \quad u_{2}=-\dot{k}_{2} e_{y} \quad \text { and } \quad u_{3}=0
$$

where $\dot{k}_{2}$ is an estimated feedback gain updated according to the following adaptative algorithm

$$
\dot{\tilde{k}}_{2}=\gamma e_{y}^{2}, \quad \tilde{k}_{2}(0)=0
$$

Then the resulting error dynamical system can be expressed as

$$
\begin{aligned}
& \dot{e}_{x}=-e_{y}-e_{z} \\
& \dot{e}_{y}=e_{x}+\left(a-\tilde{k}_{2}\right) e_{y} \\
& \dot{e}_{z}=z_{1} e_{x}+\left(x_{2}-c\right) e_{z} \\
& \dot{\tilde{k}}_{2}=\gamma e_{y}^{2}, \quad \tilde{k}_{2}(0)=0
\end{aligned}
$$

Let us consider Lyapunov function as follows

$$
V=\frac{1}{2}\left(e_{x}^{2}+e_{y}^{2}+e_{z}^{2}+\frac{1}{\gamma}\left(\tilde{k}_{2}-k_{2}^{* *}\right)^{2}\right)
$$

where $k_{2}^{* *}$ is a positive constant which will be defined later. Taking the time derivative of Eq. (20), we get,

$$
\begin{aligned}
\dot{V} & =-\left\{\left(k_{2}^{* *}-a\right) e_{y}^{2}+\left(c-x_{2}\right) e_{z}^{2}+\left(1-z_{1}\right) e_{x} e_{z}\right\} \\
& =-\left\{\left(k_{2}^{* *}-a\right) e_{y}^{2}+\left(c-s_{1}\right) e_{z}^{2}+\left(1-s_{3}\right) e_{x} e_{z}\right\} \\
& \leq 0
\end{aligned}
$$

Since $c-s_{1}>0$ and $1-s_{3}>0$ we choose $k_{1}^{* *}>a$, that is $\dot{V} \leq 0$. Similarly, since $V$ is a positive and decreasing function and $\dot{V}$ is a negative semidefinite, we can conclude that the two Rössler systems have been asymptotically synchronized under the control law (19) associated with (18).

\subsection{Third case}

The state variable $z_{1}$ of the drive system is coupled to the first equation of the response system and an external control with the state $z_{2}$ as the feedback variable is also introduced into the second equation in (11). Therefore, the feedback control law is described as

$$
u_{1}=u_{2}=0 \quad \text { and } \quad u_{3}=\tilde{k}_{3} e_{z}
$$

where $\tilde{k}_{3}$ is an estimated feedback gain updated according to the following adaptative algorithm

$$
\dot{\tilde{k}}_{3}=\gamma e_{z}^{2}, \quad \tilde{k}_{3}(0)=0
$$

Then the resulting error dynamical system can be expressed as

$$
\begin{aligned}
& \dot{e}_{x}=-e_{y}-e_{z} \\
& \dot{e}_{y}=e_{x}+a e_{y} \\
& \dot{e}_{z}=z_{1} e_{x}+\left(x_{2}-c-\tilde{k}_{3}\right) e_{z} \\
& \dot{\tilde{k}}_{3}=\gamma e_{z}^{2}, \quad \tilde{k}_{3}(0)=0
\end{aligned}
$$

Consider Lyapunov function candidate as

$$
V=\frac{1}{2}\left(e_{x}^{2}+e_{y}^{2}+e_{z}^{2}+\frac{1}{\gamma}\left(\tilde{k}_{3}-k_{3}^{* *}\right)^{2}\right)
$$

where $k_{2}^{* *}$ is a positive constant which will be defined later. Taking the time derivative of Eq. (25), we get,

$$
\begin{aligned}
\dot{V} & =-\left\{-a e_{y}^{2}+\left(k_{3}^{* *}+c-x_{2}\right) e_{z}^{2}+\left(1-z_{1}\right) e_{x} e_{z}\right\} \\
& =-\left\{-a e_{y}^{2}+\left(k_{3}^{* *}+c-s_{1}\right) e_{z}^{2}+\left(1-s_{3}\right) e_{x} e_{z}\right\} \\
& \leq 0
\end{aligned}
$$

Since $1-s_{3}>0$, and we chose $k_{1}^{* *}>c-s_{1}$, $\dot{V} \leq 0$. Similarly, since $V$ is a positive and decreasing function and $\dot{V}$ is a negative semidefinite, we can conclude that the two Rössler systems have been globally asymptotically synchronized under the control law (24) associated with (23). 


\subsection{Numerical results}

Fourth-order Runge-Kutta method is used to solve the system of differential equations. In addition, a time step size 0.001 is employed. The two parameters are chosen as $a=0.2$ and $c=5.4$ in all simulations so that the Rössler system exhibits a chaotic

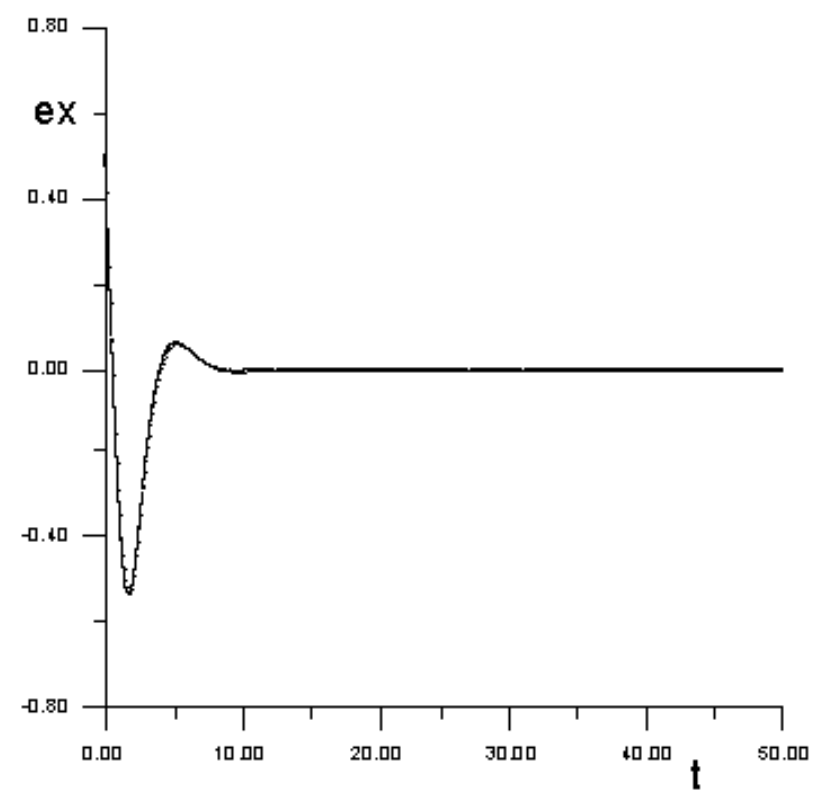

(a) behavior if no control is applied. The initial states of the drive system are $x_{1}(0)=0.5, y_{1}(0)=0.5$ and $z_{1}(0)=0.5$ and of the response system are $x_{2}(0)=1, y_{2}(0)=1$ and $z_{2}(0)=0.8$. Then $e_{x}(0)=$ $0.5, e_{y}(0)=0.5$ and $e_{z}(0)=0.3$. In this case, we assume that the drive system and the response system are two identical Rössler systems with

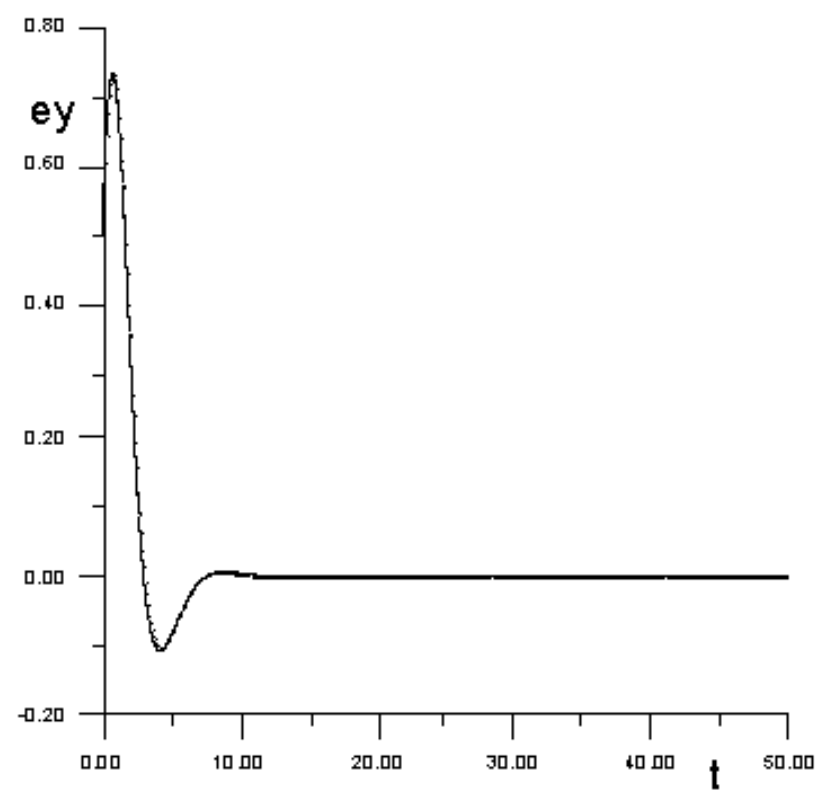

(b)

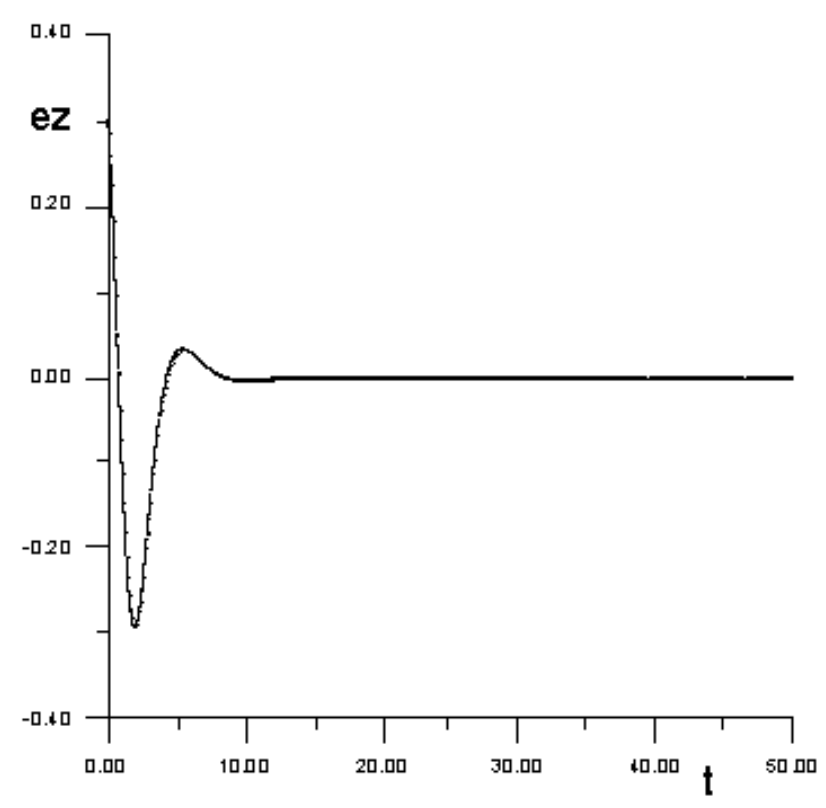

(c)

Fig. 1. Shows that the trajectory (a) $e_{x}$ of the error system tends to zero when the parameter values are $a=0.2$ and $c=5.4$ where the controls $u_{1}=\tilde{k}_{1} e_{x}$ with $\dot{\tilde{k}}_{1}=1.5 e_{x}^{2}$ are activated at $t=10$. (b) $e_{y}$ of the error system converges to zero when $a=0.2$ and $c=5.4$ where the controls $u_{1}=\tilde{k}_{1} e_{x}$ with $\dot{\tilde{k}}_{1}=1.5 e_{x}^{2}$ are activated at $t=10$. (c) $e_{z}$ of the error system converges to zero when $a=0.2$ and $c=5.4$ where the controls $u_{1}=\tilde{k}_{1} e_{x}$ with $\dot{\tilde{k}}_{1}=1.5 e_{x}^{2}$ are activated at $t=10$. 
different initial conditions. Figure 1 shows the evolutions of state synchronization errors and the history of the estimated feedback gain using the feedback control law, Eq. (13), associated with the adaptation algorithm (14). Figure 2 shows the state responses of adaptive synchronization when the adaptive control law is $u_{1}=\tilde{k}_{1} e_{x}$ with $\dot{\tilde{k}}_{1}=1.5 e_{x}^{2}$. Figure 3 shows the evolutions of state synchronization errors and the history of the estimated feedback gain using the feedback control law, Eq. (18), associated with the adaptation algorithm (19). Figure 4 shows the state responses of adaptive synchronization when the adaptive control law is $u_{2}=\tilde{k}_{2} e_{y}$ with $\dot{\tilde{k}}_{2}=e_{y}^{2}$. Figure 5 shows the evolutions of state synchronization errors and the history of the estimated feedback gain using the feedback control law, Eq. (23), associated with the adaptation algorithm (24). Figure 6 shows the state responses of adaptive synchronization when the adaptive control law is $u_{3}=\tilde{k}_{3} e_{z}$ with $\dot{\tilde{k}}_{3}=0.01 e_{z}^{2}$. These numerical results demonstrate that the Rössler systems have been asymptotically synchronized using the proposed adaptive schemes.

\section{Adaptive Synchronization of Chua's Circuit System}

Chua's circuit consists of one inductor, two capacitors, one linear resistor, and one piecewise-linear nonlinear resistor. The mathematical model equations [Matsumoto et al., 1985; Hwang et al., 1996; Hwang et al., 1997] for this circuit are

$$
\begin{aligned}
\dot{x} & =\alpha(y-x-f(x)) \\
\dot{y} & =x-y+z \\
\dot{z} & =-\beta y \\
f(x) & = \begin{cases}b x+a-b & x>1 \\
a x & |x| \leq 1 \\
b x-a+b & x<-1\end{cases}
\end{aligned}
$$

In the model equations, variables $x$ and $y$ represent the voltages across the two capacitors, and variable $z$ in the current through the inductor. Typical values of the system parameters $\alpha=9, \beta=$ $100 / 7, a=-(8 / 7)$ and $b=-(5 / 7)$ create chaotic behavior in the dynamical system (27). Through this choice, the corresponding equilibrium points of system (27) are clearly $(1.5,0,-1.5),(0,0,0)$ and $(-1.5,0,1.5)$, which are located in the regions $x>1,|x| \leq 1$ and $x<-1$, respectively.

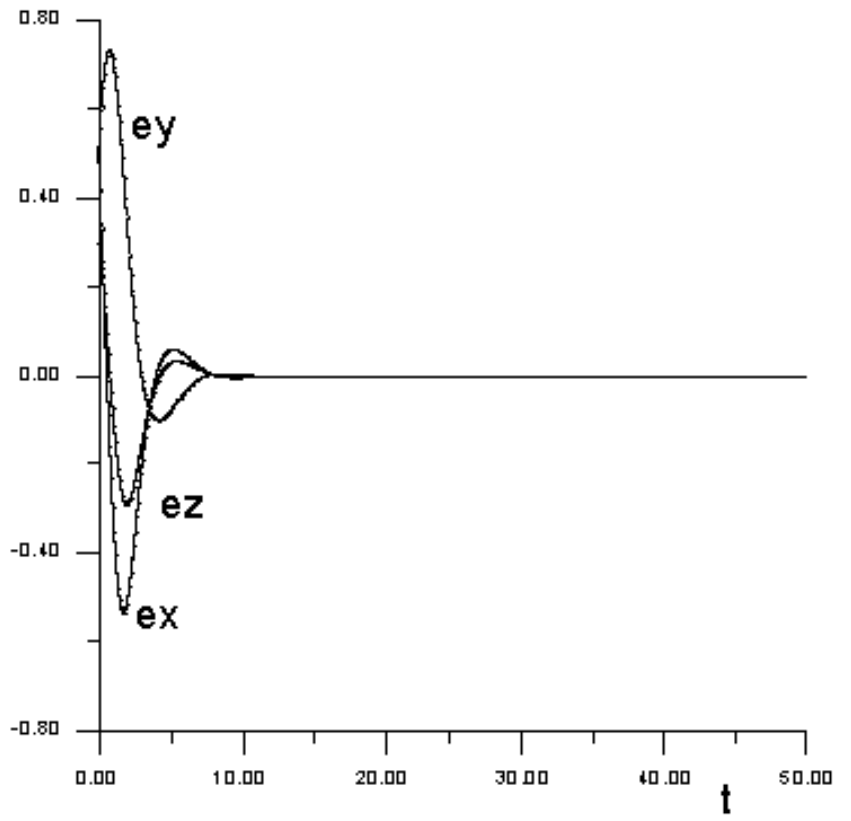

Fig. 2. Shows the state responses of adaptive synchronization when the adaptive control law is $u_{1}=\tilde{k}_{1} e_{x}$ with $\dot{\tilde{k}}_{1}=1.5 e_{x}^{2}$.

We have two Chua's circuit systems where the drive system with three state variables denoted by the subscript 1 drives the response system having identical equations denoted by the subscript 2. However, the initial condition on the drive system is different from that of the response system, therefore two Chua's circuit systems are described, respectively, by the following equations:

$$
\begin{aligned}
& \dot{x}_{1}=\alpha\left(y_{1}-x_{1}-f\left(x_{1}\right)\right) \\
& \dot{y}_{1}=x_{1}-y_{1}+z_{1} \\
& \dot{z}_{1}=-\beta y_{1}
\end{aligned}
$$

where

$$
f\left(x_{1}\right)= \begin{cases}b x_{1}+a-b & x_{1}>1 \\ a x_{1} & \left|x_{1}\right| \leq 1 \\ b x_{1}-a+b & x_{1}<1\end{cases}
$$

and

$$
\begin{aligned}
\dot{x}_{2} & =\alpha\left(y_{2}-x_{2}-f\left(x_{2}\right)\right)+u_{1} \\
\dot{y}_{2} & =x_{2}-y_{2}+z_{2}+u_{2} \\
\dot{z}_{2} & =-\beta y_{2}+u_{3} \\
f\left(x_{2}\right) & = \begin{cases}b x_{2}+a-b & x_{2}>1 \\
a x_{2} & \left|x_{2}\right| \leq 1 \\
b x_{2}-a+b & x_{2}<1\end{cases}
\end{aligned}
$$




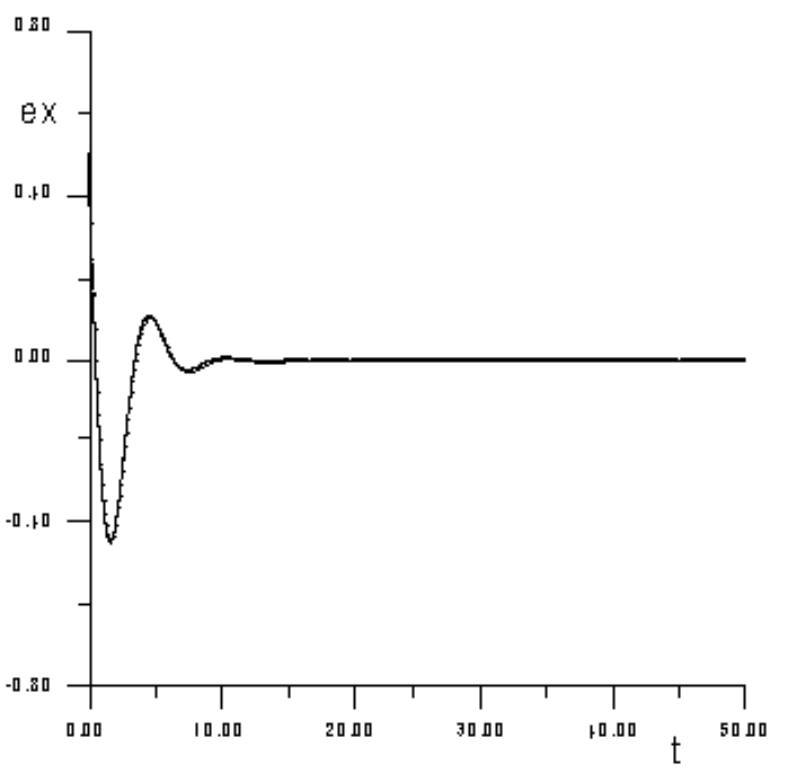

(a)

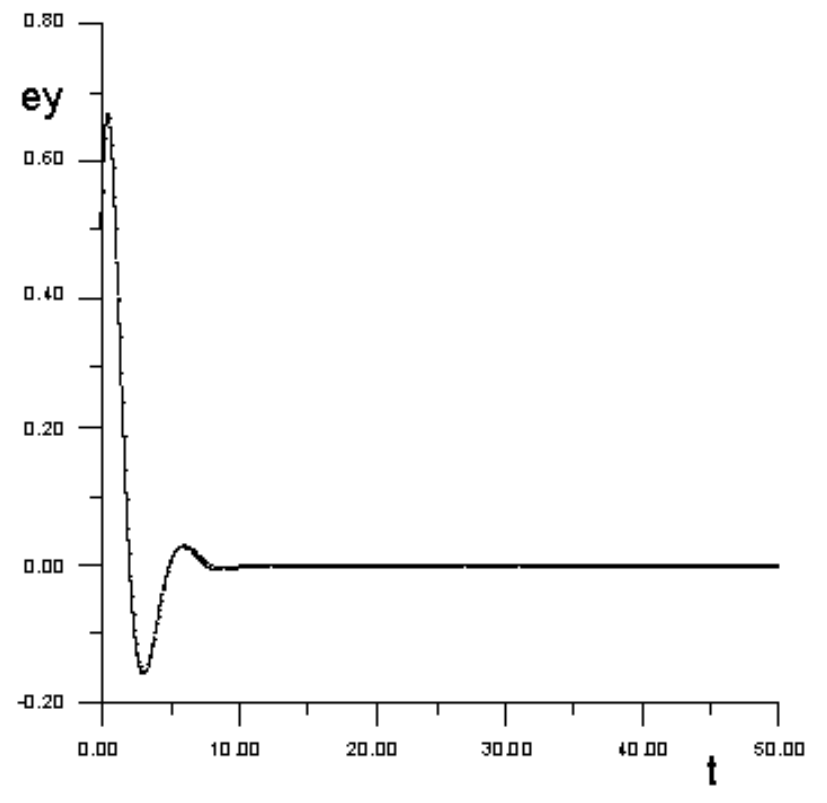

(b)

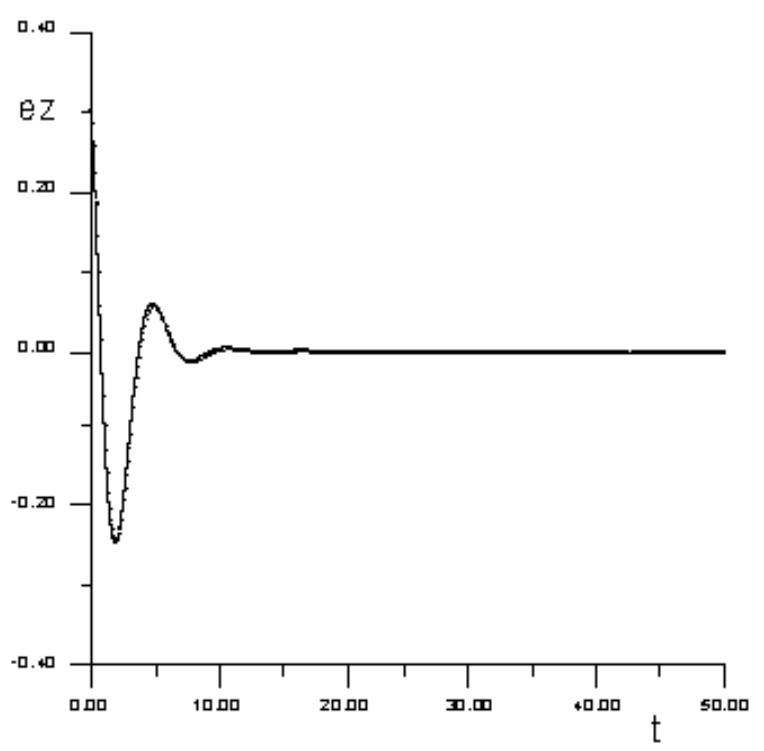

(c)

Fig. 3. Shows that the trajectory (a) $e_{x}$ of the error system converges to zero when $a=0.2$ and $c=5.4$ where the controls $u_{2}=\tilde{k}_{2} e_{y}$ with $\dot{\tilde{k}}_{2}=e_{y}^{2}$ are activated at $t=15$. (b) $e_{y}$ of the error system converges to zero when $a=0.2$ and $c=5.4$ where the controls $u_{2}=\tilde{k}_{2} e_{y}$ with $\dot{\tilde{k}}_{2}=e_{y}^{2}$ are activated at $t=10$. (c) $e_{z}$ of the error system converges to zero when $a=0.2$ and $c=5.4$ where the controls $u_{2}=\tilde{k}_{2} e_{y}$ with $\dot{\tilde{k}}_{2}=e_{y}^{2}$ are activated at $t=18$.

We have introduced three control inputs, $u_{1}$, $u_{2}$ and $u_{3}$ in Eq. (29). $u_{1}, u_{2}$ and $u_{3}$ are to be determined for the purpose of synchronizing the two identical Chua's circuit systems with the same but unknown parameters $a, b$ and $c$ in spite of the differences in initial conditions.

Remark 2. Chua's circuit system has a bounded, zero volume, globally attracting set [Matsumoto et al., 1995; Hwang et al., 1997]. Therefore, the state trajectories $x(t), y(t)$ and $z(t)$ are globally bounded for all $t \geq 0$ and continuously differentiable with respect to time. Consequently, there exist three positive constants $s_{1}, s_{2}$ and $s_{3}$ such that $|x(t)| \leq s_{1}<\infty,|y(t)| \leq s_{2}<\infty$ and $|z(t)| \leq s_{3}<\infty$ hold for all $t \geq 0$.

Let us define the state errors between the 


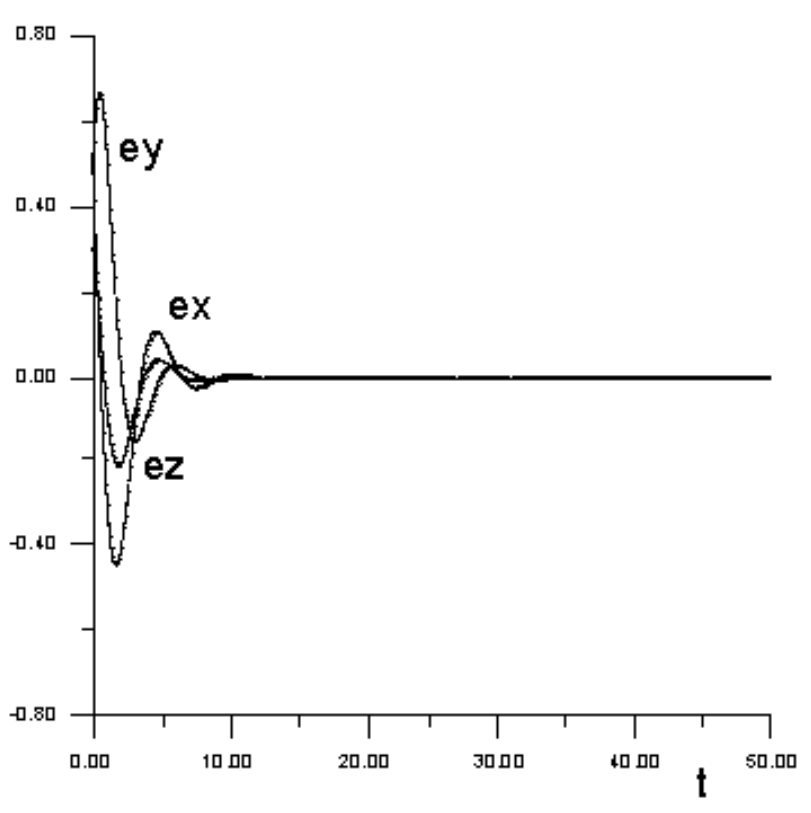

Fig. 4. Shows the state responses of adaptive synchronization when the adaptive control law is $u_{2}=\tilde{k}_{2} e_{y}$ with $\dot{\tilde{k}}_{2}=e_{y}^{2}$.

response system that is to be controlled and the controlling drive system as

$$
e_{x}=x_{2}-x_{1}, \quad e_{y}=y_{2}-y_{1} \quad \text { and } \quad e_{z}=z_{2}-z_{1}
$$

Subtracting Eq. (28) from Eq. (29) and using the notation (30) yields

$$
\begin{aligned}
& \dot{e}_{x}=\alpha\left(e_{y}-e_{x}-g\left(e_{x}\right)\right)+u_{1} \\
& \dot{e}_{y}=e_{x}-e_{y}+e_{z}+u_{2} \\
& \dot{e}_{z}=-\beta e_{y}+u_{3} \\
& g\left(e_{x}\right)= \begin{cases}a e_{x} & \left|e_{x}\right| \leq 2 \\
b e_{x} & \text { otherwise }\end{cases}
\end{aligned}
$$

hence the synchronization problem is now replaced by the equivalent problem of stabilizing the system
(32) using a suitable choice of the control laws $u_{1}$, $u_{2}$ and $u_{3}$. Let us now discuss the following three cases of control inputs $u_{1}, u_{2}$ and $u_{3}$ :

\subsection{First case}

The state variable $x_{1}$ of the drive system is coupled to the first equation of the response system and an external control with the state $x_{2}$ as the feedback variable is introduced into the first equation in (32). Therefore, the feedback control law is described as

$$
u_{1}=-\tilde{k}_{1} e_{x}, \quad u_{2}=0 \quad \text { and } \quad u_{3}=0
$$

where $\tilde{k}_{1}$ denotes an estimated feedback gain which is updated according to the following adaptative algorithm

$$
\dot{\tilde{k}}_{1}=\gamma e_{x}^{2}, \quad \tilde{k}_{1}(0)=0
$$

Then the resulting error dynamical system can be expressed as

$$
\begin{aligned}
& \dot{e}_{x}=\alpha\left(e_{y}-\left(1+\tilde{k}_{1}\right) e_{x}-g\left(e_{x}\right)\right) \\
& \dot{e}_{y}=e_{x}-e_{y}+e_{z} \\
& \dot{e}_{z}=-\beta e_{y} \\
& \dot{\tilde{k}}_{1}=\gamma e_{x}^{2}, \quad \tilde{k}_{1}(0)=0
\end{aligned}
$$

where

$$
g\left(e_{x}\right)= \begin{cases}a e_{x} & \left|e_{x}\right| \leq 2 \\ b e_{x} & \text { otherwise }\end{cases}
$$

Let us consider the Lyapunov function $V$ which is defined by

$$
V=\frac{1}{2}\left(\frac{1}{\alpha} e_{x}^{2}+e_{y}^{2}+e_{z}^{2}+\frac{1}{\gamma}\left(\tilde{k}_{1}-k_{1}^{* *}\right)^{2}\right)
$$

where $k_{1}^{* *}$ is a positive constant which will be defined later. Taking the time derivative of Eq. (35), then we get

$$
\begin{aligned}
\dot{V} & =-\left\{\left(1+l+k_{1}^{* *}\right) e_{x}^{2}+e_{y}^{2}-2 e_{x} e_{y}+(\beta-1) e_{y} e_{z}\right\} \\
& =-\left[\begin{array}{lll}
\left|e_{x}\right| & \left|e_{y}\right| & \left|e_{z}\right|
\end{array}\right]\left[\begin{array}{ccc}
\left(1+l+k_{1}^{* *}\right) & 0 & 0 \\
-2 & 1 & 2(\beta-1) \\
0 & -(\beta-1) & 0
\end{array}\right]\left[\begin{array}{c}
\left|e_{x}\right| \\
\left|e_{y}\right| \\
\left|e_{z}\right|
\end{array}\right] \\
& \equiv-\left[\begin{array}{lll}
\left|e_{x}\right| & \left|e_{y}\right| & \left|e_{z}\right|
\end{array}\right] \Psi\left(k_{1}^{* *}\right)\left[\begin{array}{lll}
\left|e_{x}\right| & \left|e_{y}\right| & \left|e_{z}\right|
\end{array}\right]^{T}
\end{aligned}
$$




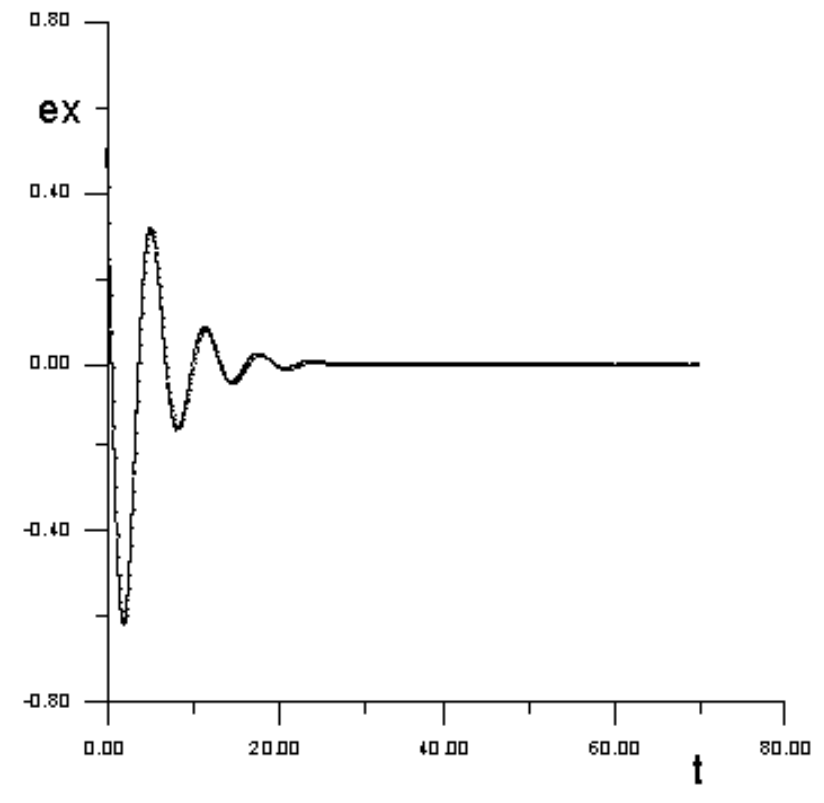

(a)

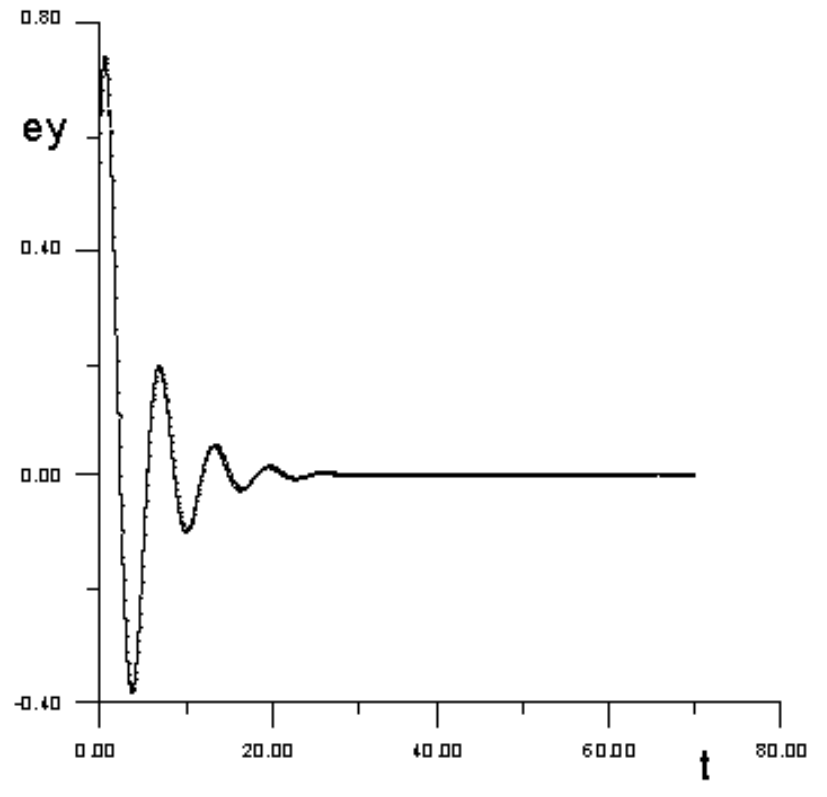

(b)

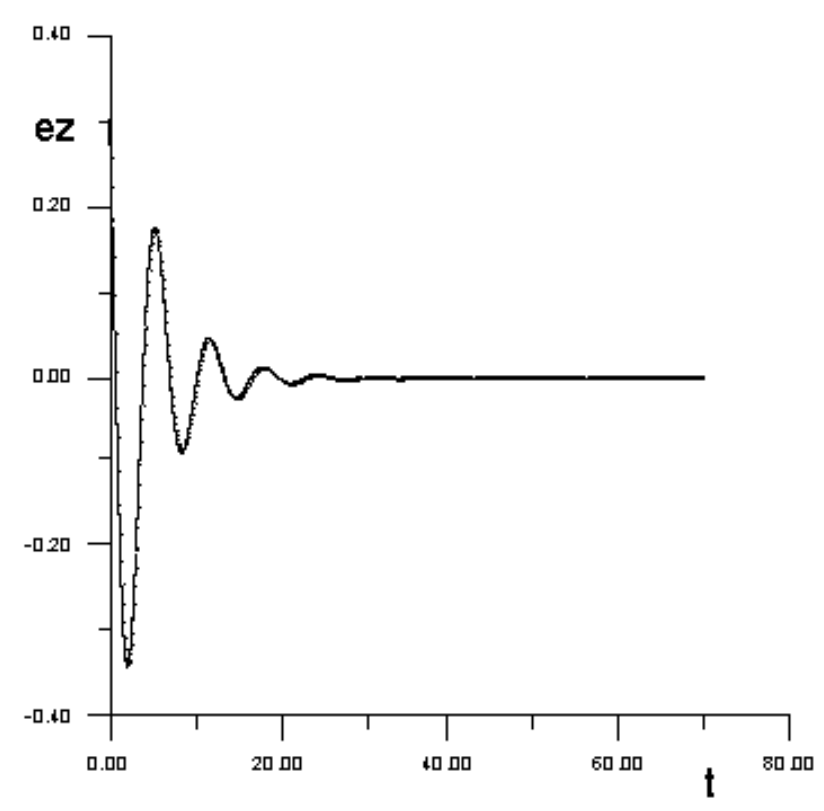

(c)

Fig. 5. Shows that the trajectory (a) $e_{x}$ of the error system converges to zero when $a=0.2$ and $c=5.4$ where the controls $u_{3}=\tilde{k}_{3} e_{z}$ with $\dot{\tilde{k}}_{3}=0.01 e_{z}^{2}$ are activated at $t=25$. (b) $e_{y}$ of the error system converges to zero when $a=0.2$ and $c=5.4$ where the controls $u_{3}=\tilde{k}_{3} e_{z}$ with $\dot{\tilde{k}}_{3}=0.01 e_{z}^{2}$ are activated at $t=25$. (c) $e_{z}$ of the error system converges to zero when $a=0.2$ and $c=5.4$ where the controls $u_{3}=\tilde{k}_{3} e_{z}$ with $\dot{\tilde{k}}_{3}=0.01 e_{z}^{2}$ are activated after $t>35$.

where

$$
l= \begin{cases}a & \left|e_{x}\right| \leq 2 \\ b & \text { otherwise }\end{cases}
$$

If $k_{1}^{* *}$ is appropriately chosen such that the $3 \times 3$ matrix $\Psi\left(k_{1}^{* *}\right)$ in Eq. (36) is positive definite, then $\dot{V} \leq 0$ holds. Since $V$ is a positive and decreas- ing function, $\dot{V}$ is a negative semidefinite. It follows that the equilibrium point $\left(e_{x}=0, e_{y}=0\right.$, $\left.e_{z}=0, \tilde{k}_{1}=k_{1}^{* *}\right)$ of the system (34) is uniformly stable, i.e. $e_{x}(t), e_{y}(t), e_{z}(t) \in L_{\infty}$ and $\tilde{k}_{1}(t) \in L_{\infty}$. From Eq. (36) we can easily show that the squares of $e_{x}(t), e_{y}(t)$ and $e_{z}(t)$ are integrable with respect to time $t$, i.e. $e_{x}(t), e_{y}(t), e_{z}(t) \in L_{2}$. Next by 


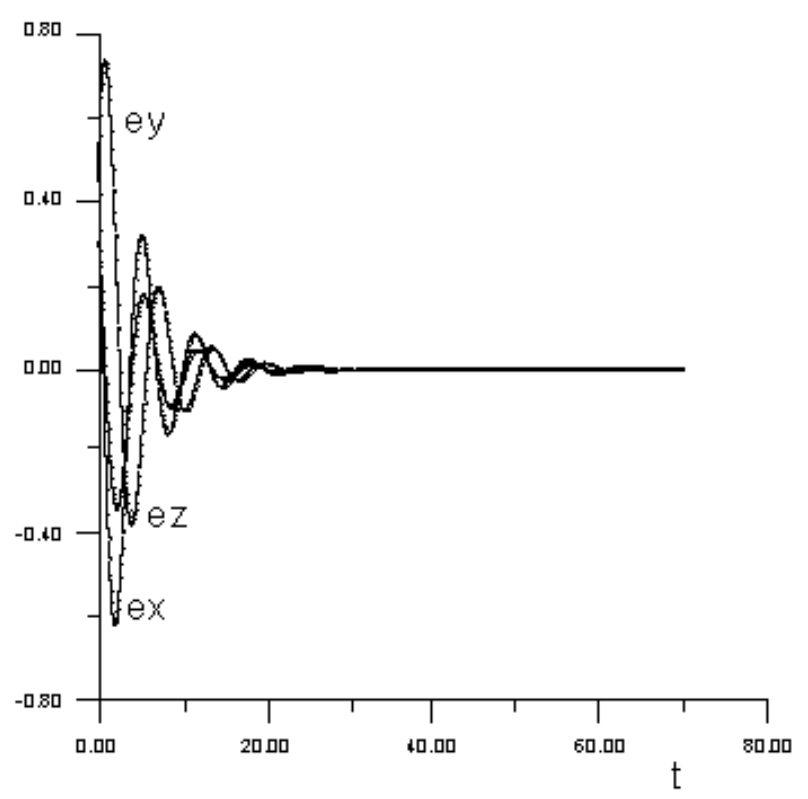

Fig. 6. Shows the state responses of adaptive synchronization when the adaptive control law is $u_{3}=\tilde{k}_{3} e_{z}$ with $\dot{\tilde{k}}_{3}=0.01 e_{z}^{2}$.

Barbalat's Lemma Eq. (34) implies that $\dot{e}_{x}(t), \dot{e}_{y}(t)$, $\dot{e}_{z}(t) \in L_{\infty}$, which in turn implies $e_{x}(t), e_{y}(t)$, $e_{z}(t) \rightarrow 0$ as $t \rightarrow \infty$. Thus, in the closed-loop system $x_{2}(t) \rightarrow x_{1}(t), y_{2}(t) \rightarrow y_{1}(t), z_{2}(t) \rightarrow z_{1}(t)$ and $t \rightarrow \infty$. This implies that the two Chua's circuit systems have been globally asymptotically synchronized under the control law (34) associated with (33).

\subsection{Second case}

The state variable $y_{1}$ of the drive system is coupled to the first equation of the response system and an external control with the state $y_{2}$ as the feedback variable is also introduced into the second equation in (32). Therefore, the feedback control law is described as

$$
u_{1}=0, \quad u_{2}=-\tilde{k}_{2} e_{y} \quad \text { and } \quad u_{3}=0
$$

where $\tilde{k}_{2}$ is an estimated feedback gain updated according to the following algorithm

$$
\dot{\tilde{k}}_{2}=\gamma e_{y}^{2}, \quad \tilde{k}_{2}(0)=0
$$

Then the resulting error dynamical system can be expressed as

$$
\begin{aligned}
& \dot{e}_{x}=\alpha\left(e_{y}-e_{x}-g\left(e_{x}\right)\right) \\
& \dot{e}_{y}=e_{x}-\left(1+\tilde{k}_{2}\right) e_{y}+e_{z} \\
& \dot{e}_{z}=-\beta e_{y} \\
& \dot{\tilde{k}}_{2}=\gamma e_{y}^{2}, \quad \tilde{k}_{2}(0)=0
\end{aligned}
$$

where

$$
g\left(e_{x}\right)= \begin{cases}a e_{x} & \left|e_{x}\right| \leq 2 \\ b e_{x} & \text { otherwise }\end{cases}
$$

Let us consider Lyapunov function by

$$
V=\frac{1}{2}\left(\frac{1}{\alpha} e_{x}^{2}+e_{y}^{2}+e_{z}^{2}+\frac{1}{\gamma}\left(\tilde{k}_{2}-k_{2}^{* *}\right)^{2}\right)
$$

where $k_{2}^{* *}$ is a positive constant which will be defined later. Taking the time derivative of Eq. (40), then we get,

$$
\begin{aligned}
41 \dot{V} & =-\left\{(1+l) e_{x}^{2}+\left(1+k_{2}^{* *}\right) e_{y}^{2}-2 e_{x} e_{y}+(\beta-1) e_{y} e_{z}\right\} \\
& =-\left[\begin{array}{lll}
\left|e_{x}\right| & \left|e_{y}\right| & \left|e_{z}\right|
\end{array}\right]\left[\begin{array}{ccc}
(1+l) & -1 & 0 \\
-1 & 1+k_{2}^{* *} & -(\beta-1) \\
0 & 2(\beta-1) & 0
\end{array}\right]\left[\begin{array}{c}
\left|e_{x}\right| \\
\left|e_{y}\right| \\
\left|e_{z}\right|
\end{array}\right] \\
& \equiv-\left[\begin{array}{lll}
\left|e_{x}\right| & \left|e_{y}\right| & \left|e_{z}\right|
\end{array}\right] \Psi\left(k_{1}^{* *}\right)\left[\begin{array}{lll}
\left|e_{x}\right| & \left|e_{y}\right| & \left|e_{z}\right|
\end{array}\right]^{T}
\end{aligned}
$$

where

$$
l= \begin{cases}a & \left|e_{x}\right| \leq 2 \\ b & \text { otherwise }\end{cases}
$$

If $k_{2}^{* *}$ is appropriately chosen such that the $3 \times 3$ matrix $\Phi\left(k_{2}^{* *}\right)$ in Eq. (41) is positive definite, then $\dot{V} \leq 0$ holds. Similarly, since $V$ is a positive and decreasing function and $\dot{V}$ is a negative semidefinite, we can conclude that the two Chua's circuit systems have been globally asymptotically synchronized under the control law (39) associated with (38).

\subsection{Third case}

The state variable $z_{1}$ of the drive system is coupled to the first equation of the response system and an 
external control with the state $z_{2}$ as the feedback variable is also introduced into the second equation in (32). Therefore, the feedback control law is described as

$$
u_{1}=u_{2}=0 \quad \text { and } \quad u_{3}=\tilde{k}_{3} e_{z}
$$

where $\tilde{k}_{3}$ is an estimated feedback gain updated according to the following adaptative algorithm

$$
\dot{\tilde{k}}_{3}=\gamma e_{z}^{2}, \quad \tilde{k}_{3}(0)=0
$$

Then the resulting error dynamical system can be expressed as

$$
\begin{aligned}
& \dot{e}_{x}=\alpha\left(e_{y}-e_{x}-g\left(e_{x}\right)\right) \\
& \dot{e}_{y}=e_{x}-e_{y}+e_{z} \\
& \dot{e}_{z}=-\beta e_{y}-\tilde{k}_{3} e_{z} \\
& \dot{\tilde{k}}_{3}=\gamma e_{z}^{2}, \quad \tilde{k}_{3}(0)=0
\end{aligned}
$$

where

$$
g\left(e_{x}\right)= \begin{cases}a e_{x} & \left|e_{x}\right| \leq 2 \\ b e_{x} & \text { otherwise }\end{cases}
$$

Consider Lyapunov function candidate as

$$
V=\frac{1}{2}\left(\frac{1}{\alpha} e_{x}^{2}+e_{y}^{2}+e_{z}^{2}+\frac{1}{\gamma}\left(\tilde{k}_{3}-k_{3}^{* *}\right)^{2}\right)
$$

where $k_{2}^{* *}$ is a positive constant which will be defined later. Taking the time derivative of Eq. (45), we get,

$$
\begin{aligned}
\dot{V} & =-\left\{(1+l) e_{x}^{2}+e_{y}^{2}+k_{3}^{* *} e_{z}^{2}-2 e_{x} e_{y}+(\beta-1) e_{y} e_{z}\right\} \\
& =-\left[\begin{array}{lll}
\left|e_{x}\right| & \left|e_{y}\right| & \left|e_{z}\right|
\end{array}\right]\left[\begin{array}{ccc}
(1+l) & 0 & 0 \\
-2 & 1 & \beta-1 \\
0 & 0 & k_{3}^{* *}
\end{array}\right]\left[\begin{array}{c}
\left|e_{x}\right| \\
\left|e_{y}\right| \\
\left|e_{z}\right|
\end{array}\right] \\
& \equiv-\left[\begin{array}{lll}
\left|e_{x}\right| & \left|e_{y}\right| & \left|e_{z}\right|
\end{array}\right] \Omega\left(k_{3}^{* *}\right)\left[\begin{array}{lll}
\left|e_{x}\right| & \left|e_{y}\right| & \left|e_{z}\right|
\end{array}\right]^{T}
\end{aligned}
$$

where

$$
l= \begin{cases}a & \left|e_{x}\right| \leq 2 \\ b & \text { otherwise }\end{cases}
$$

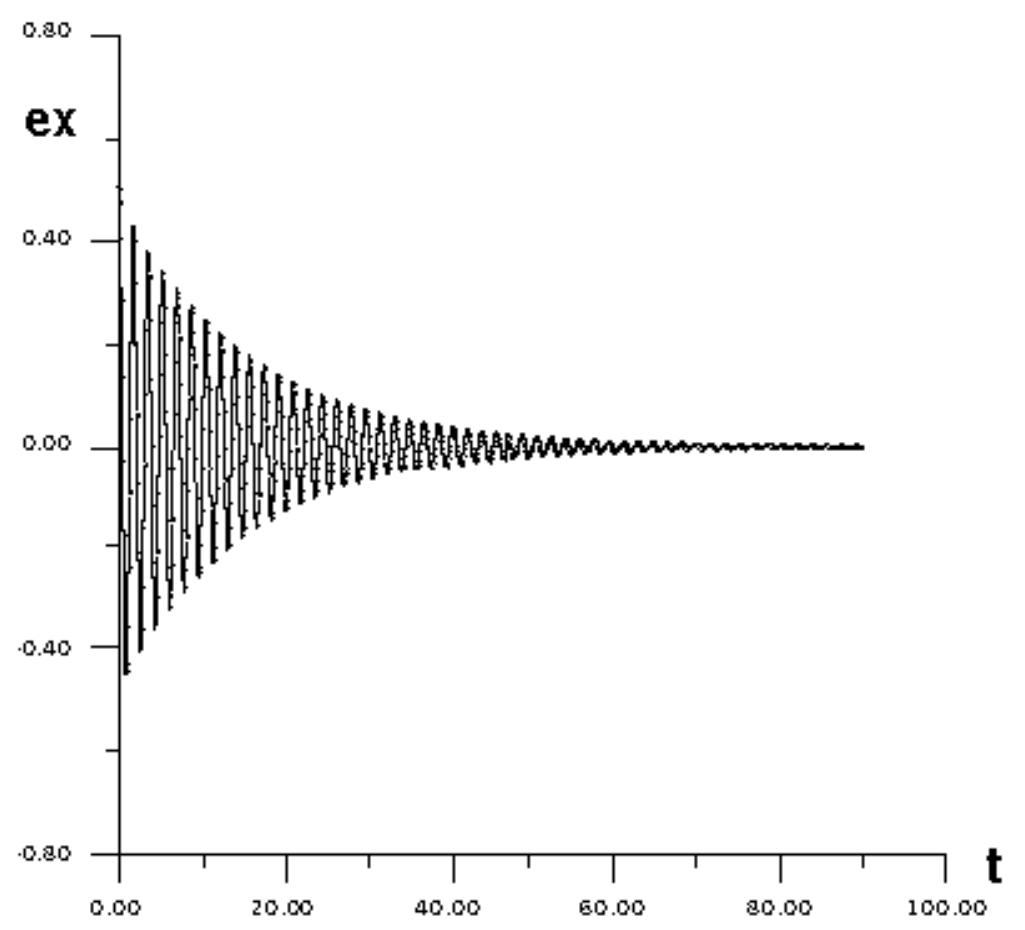

(a)

Fig. 7. Shows that the trajectory (a) $e_{x}$ of the error system tends to zero when the parameter values are $\alpha=9, \beta=100 / 7$, $a=-(8 / 7)$ and $b=-(5 / 7)$ where the controls $u_{1}=\tilde{k}_{1} e_{x}$ with $\dot{\tilde{k}}_{1}=5 e_{x}^{2}$ are activated after $t>40$. (b) $e_{y}$ of the error system converges to zero when $\alpha=9, \beta=100 / 7, a=-(8 / 7)$ and $b=-(5 / 7)$ where the controls $u_{1}=\tilde{k}_{1} e_{x}$ with $\dot{\tilde{k}}_{1}=5 e_{x}^{2}$ are activated after $t>40$. (c) $e_{z}$ of the error system converges to zero when $\alpha=9, \beta=100 / 7, a=-(8 / 7)$ and $b=-(5 / 7)$ where the controls $u_{1}=\tilde{k}_{1} e_{x}$ with $\dot{\tilde{k}}_{1}=5 e_{x}^{2}$ are activated after $t>40$. 


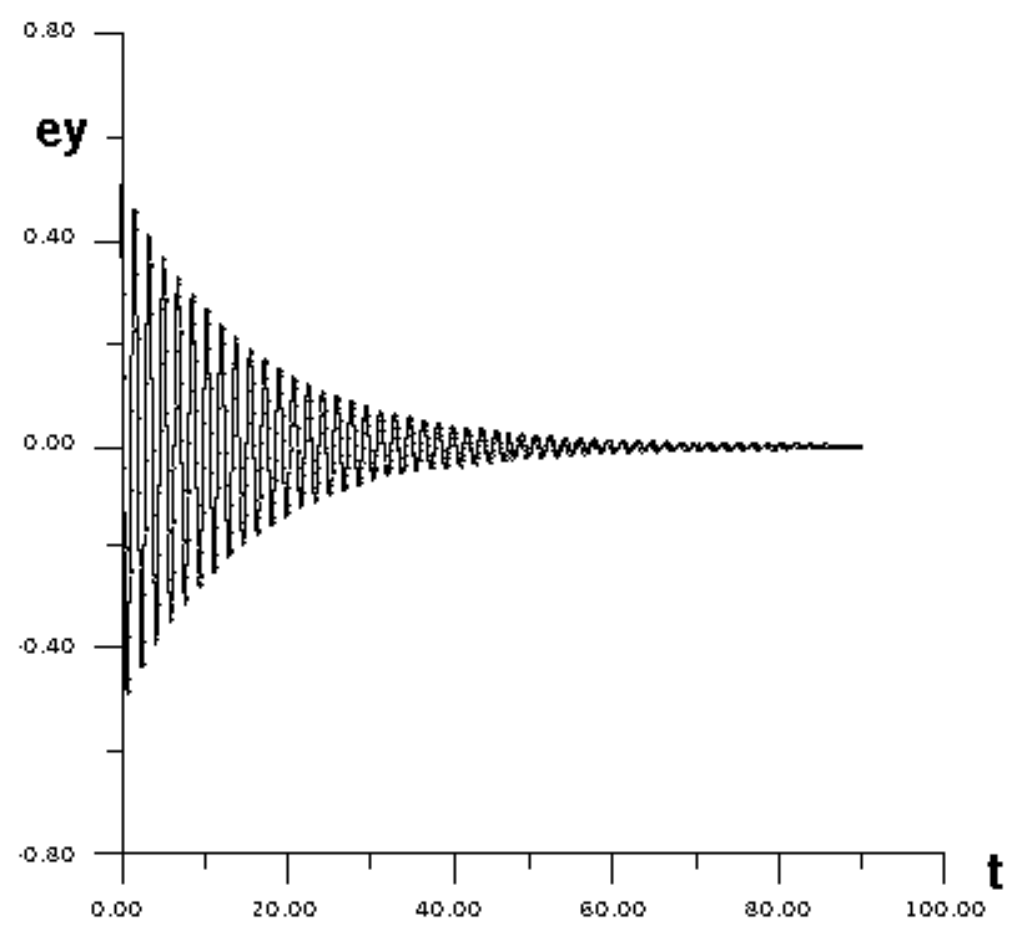

(b)

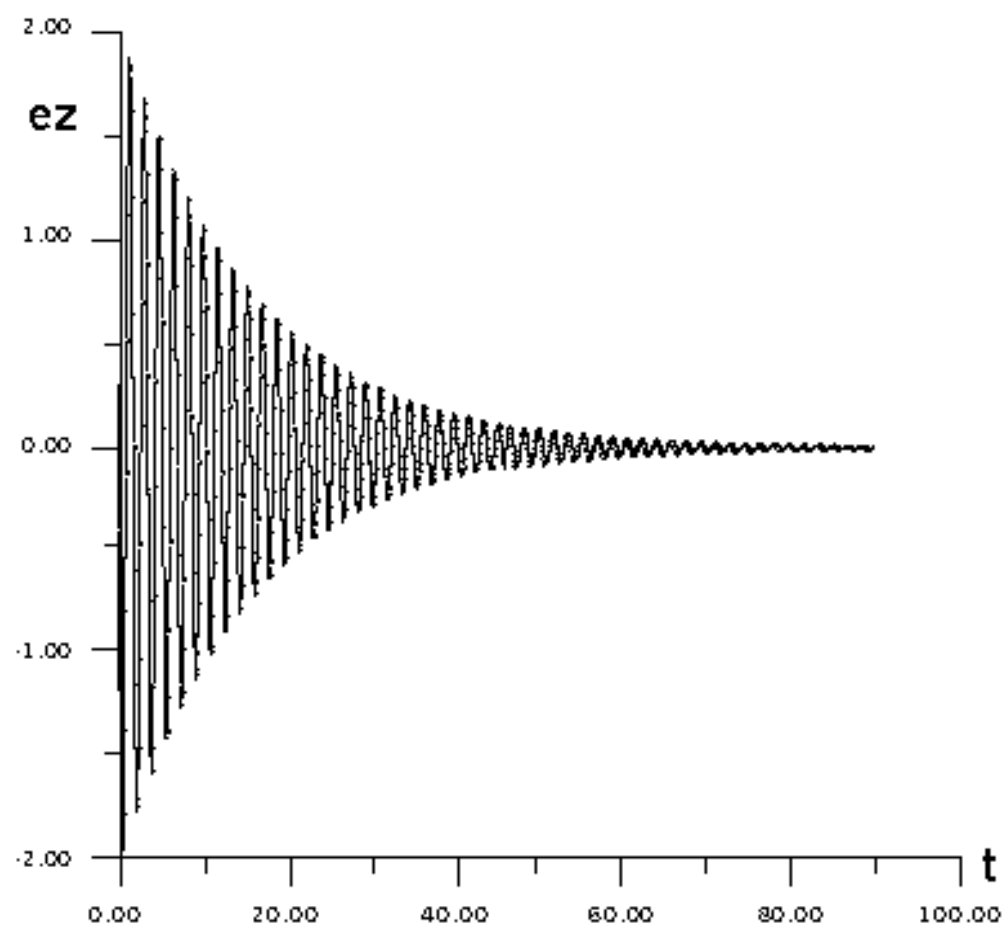

(c)

Fig. 7. (Continued) 
If $k_{2}^{* *}$ is appropriately chosen such that the $3 \times 3$ matrix $\Omega\left(k_{3}^{* *}\right)$ in Eq. (46) is positive definite, then $\dot{V} \leq 0$ holds. Similarly, since $V$ is a positive and decreasing function and $\dot{V}$ is a negative semidefinite, we can conclude that the two Chua's circuit systems have been globally asymptotically synchronized under the control law (45) associated with (44).

\subsection{Numerical results}

Fourth-order Runge-Kutta method is used to solve the system of differential equations. In addition, a time step size 0.001 is employed. The three parameters are chosen as $\alpha=9, \beta=100 / 7, a=-(8 / 7)$ and $b=-(5 / 7)$ in all simulations so that the Chua's circuit system exhibits chaotic behavior if no control is applied. The initial states $x_{1}(0)=0.5$, $y_{1}(0)=0.5$ and $z_{1}(0)=-0.5$ of the drive system and $x_{2}(0)=1, y_{2}(0)=1, z_{1}(0)=-0.2$ of the response system. Then $e_{x}(0)=0.5, e_{y}(0)=0.5$ and $e_{z}(0)=0.3$. In this case, we assume that the drive system and the response system are two identical Chua's circuit systems with different ini- tial conditions. Figure 7 shows the evolutions of state synchronization errors and the history of the estimated feedback gain using the feedback control law, Eq. (33), associated with the adaptation algorithm (34). Figure 8 shows the state responses of adaptive synchronization when the adaptive control law is $u_{1}=\tilde{k}_{1} e_{x}$ with $\dot{\tilde{k}}_{1}=5 e_{x}^{2}$. Figure 9 shows the evolutions of state synchronization errors and the history of the estimated feedback gain using the feedback control law, Eq. (38), associated with the adaptation algorithm (39). Figure 10 shows the state responses of adaptive synchronization when the adaptive control law is $u_{2}=\tilde{k}_{2} e_{y}$ with $\dot{\tilde{k}}_{2}=4 e_{y}^{2}$. Figure 11 shows the evolutions of state synchronization errors and the history of the estimated feedback gain using the feedback control law, Eq. (44), associated with the adaptation algorithm (45). Figure 12 shows the state responses of adaptive synchronization when the adaptive control law is $u_{3}=\tilde{k}_{3} e_{z}$ with $\dot{\tilde{k}}_{3}=3 e_{z}^{2}$. These numerical results demonstrate that the Chua's circuit systems have been asymptotically synchronized using the proposed adaptive schemes.

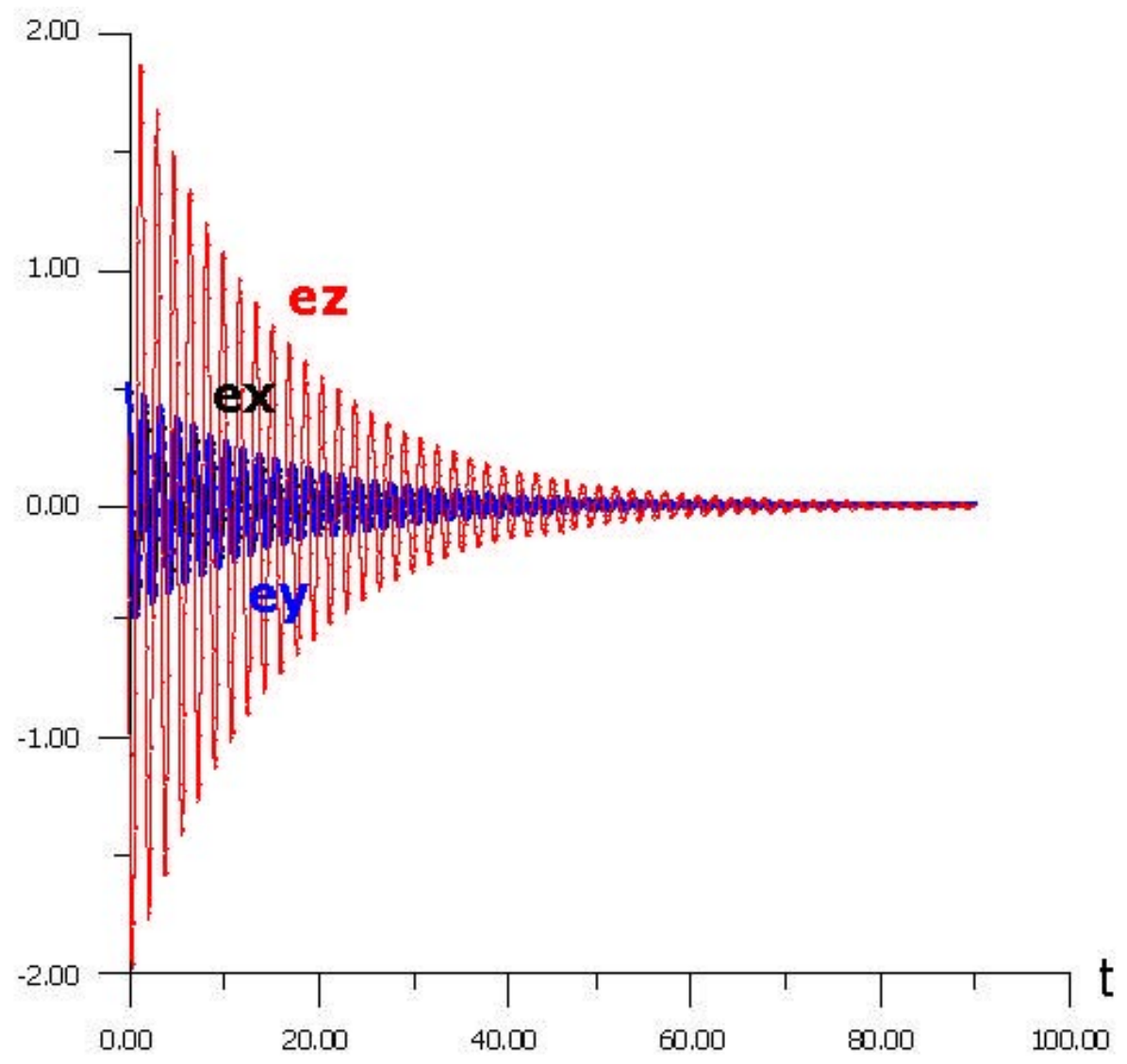

Fig. 8. Shows the state responses of adaptive synchronization when the adaptive control law is $u_{1}=\tilde{k}_{1} e_{x}$ with $\dot{\tilde{k}}_{1}=5 e_{x}^{2}$. 


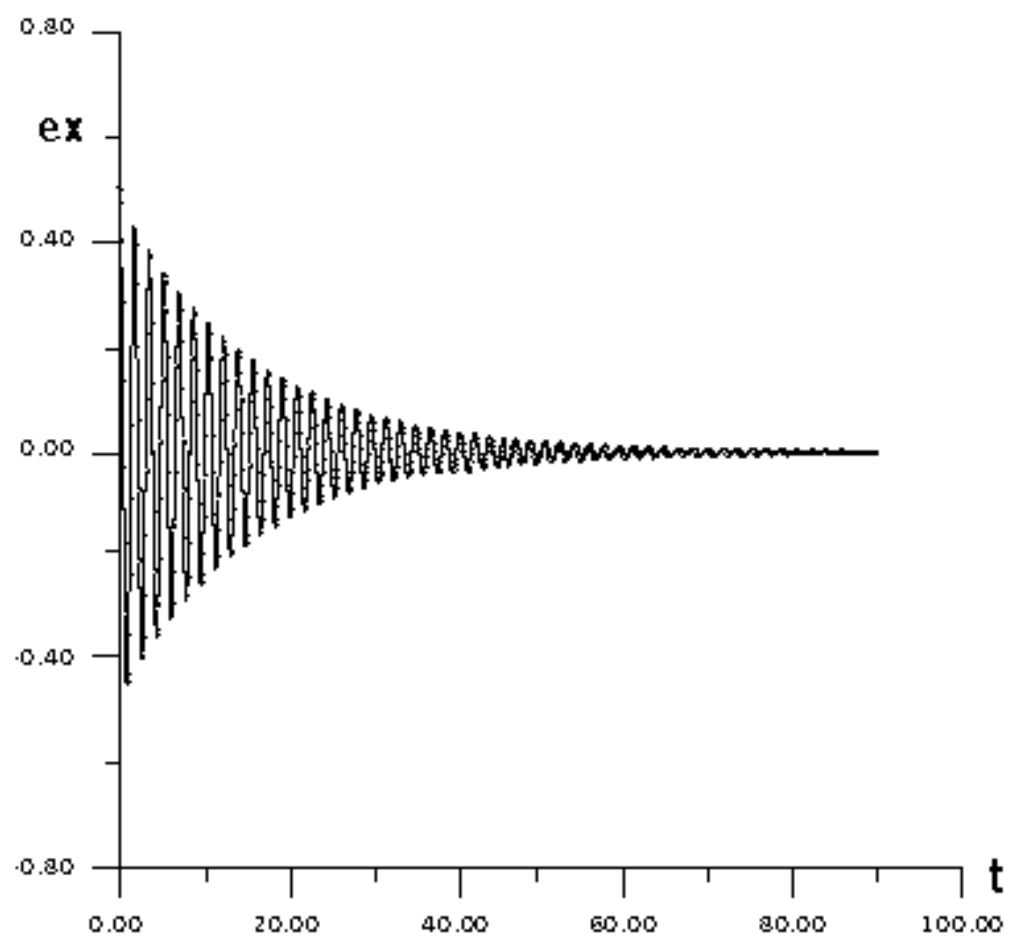

(a)

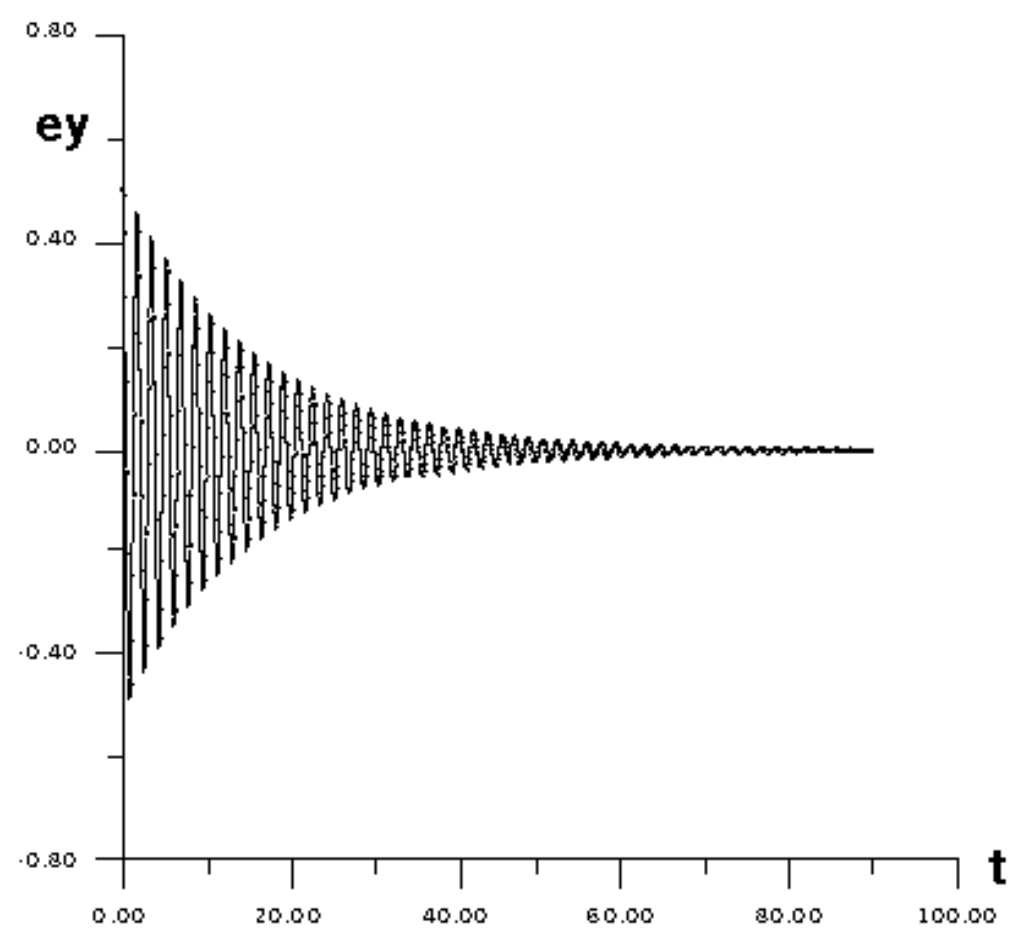

(b)

Fig. 9. Shows that the trajectory (a) $e_{x}$ of the error system converges to zero when $\alpha=9, \beta=100 / 7, a=-(8 / 7)$ and $b=-(5 / 7)$ where the controls $u_{2}=\tilde{k}_{2} e_{y}$ with $\dot{\tilde{k}}_{2}=4 e_{y}^{2}$ are activated at $t=15$. (b) $e_{y}$ of the error system converges to zero when $\alpha=9, \beta=100 / 7, a=-(8 / 7)$ and $b=-(5 / 7)$ where the controls $u_{2}=\tilde{k}_{2} e_{y}$ with $\dot{\tilde{k}}_{2}=4 e_{y}^{2}$ are activated at $t=15$. (c) $e_{z}$ of the error system converges to zero when $\alpha=9, \beta=100 / 7, a=-(8 / 7)$ and $b=-(5 / 7)$ where the controls $u_{2}=\tilde{k}_{2} e_{y}$ with $\dot{\tilde{k}}_{2}=4 e_{y}^{2}$ are activated at $t=15$. 
1594 A. S. Hegazi et al.

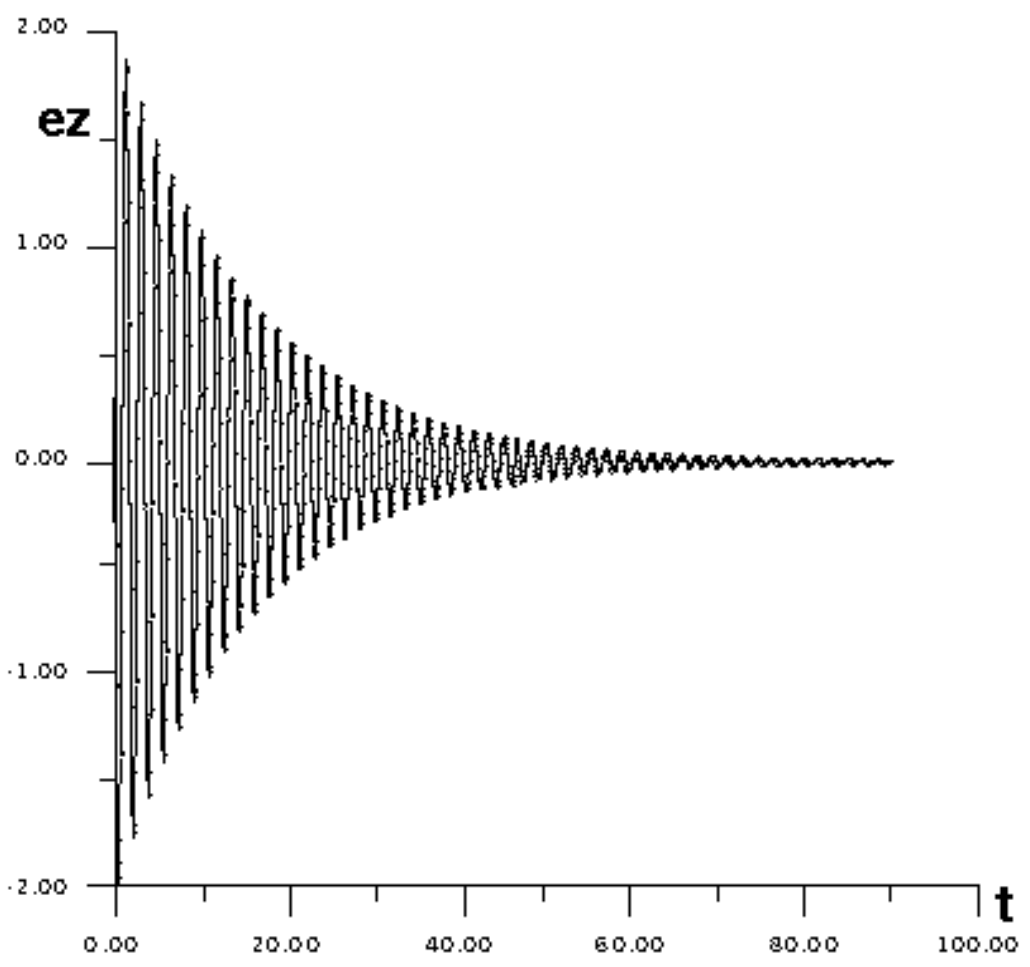

(c)

Fig. 9. (Continued)

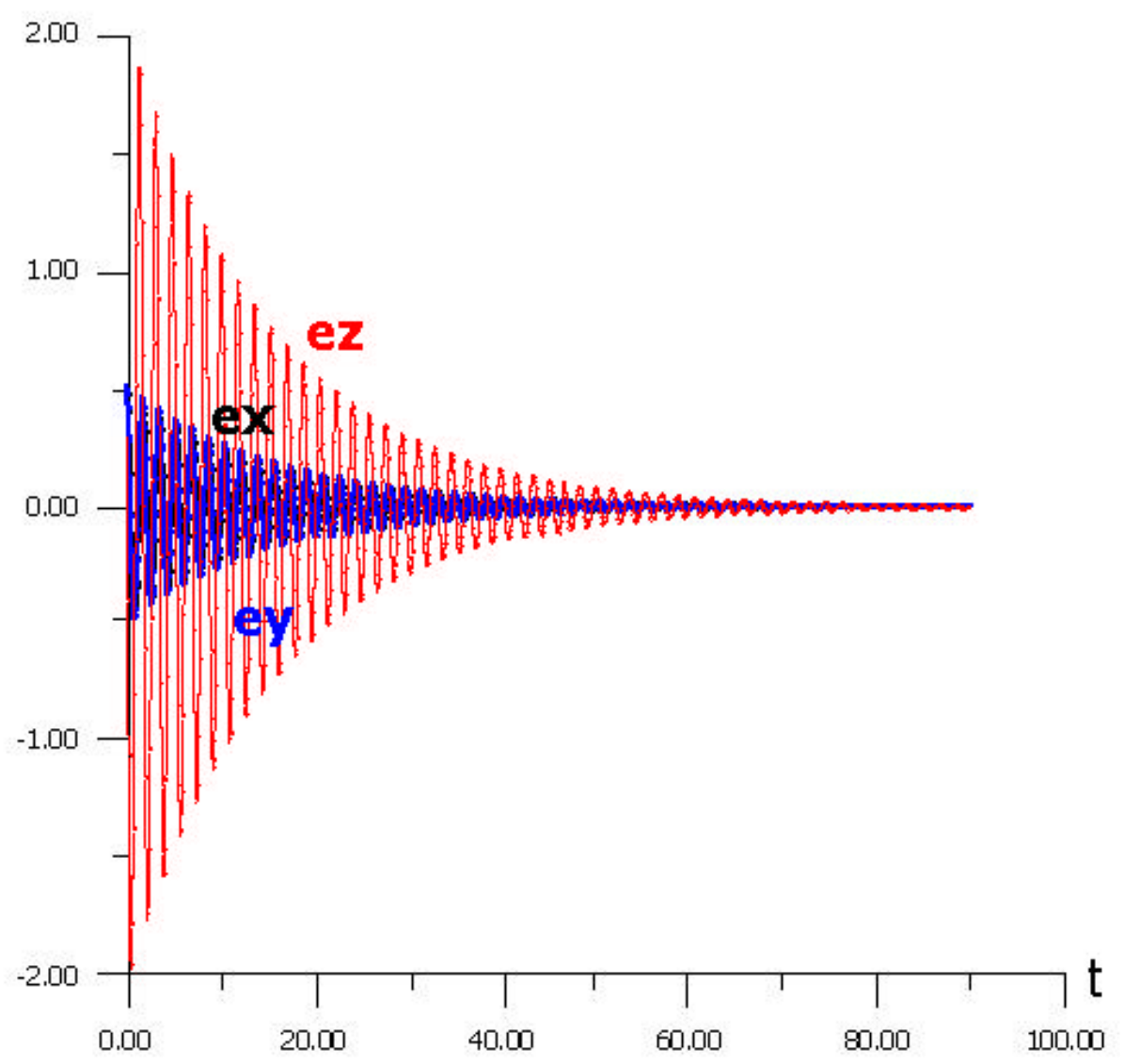

Fig. 10. Shows the state responses of adaptive synchronization when the adaptive control law is $u_{2}=\tilde{k}_{2} e_{y}$ with $\dot{\tilde{k}}_{2}=4 e_{y}^{2}$. 


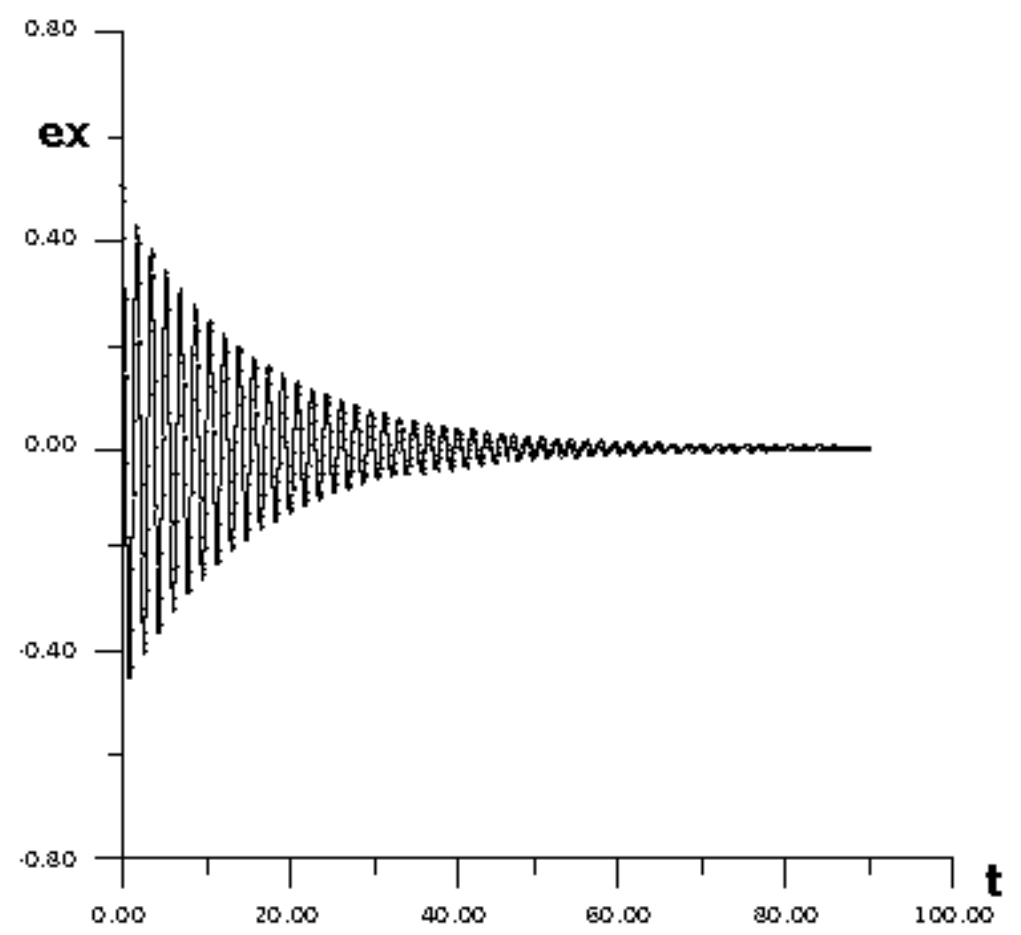

(a)

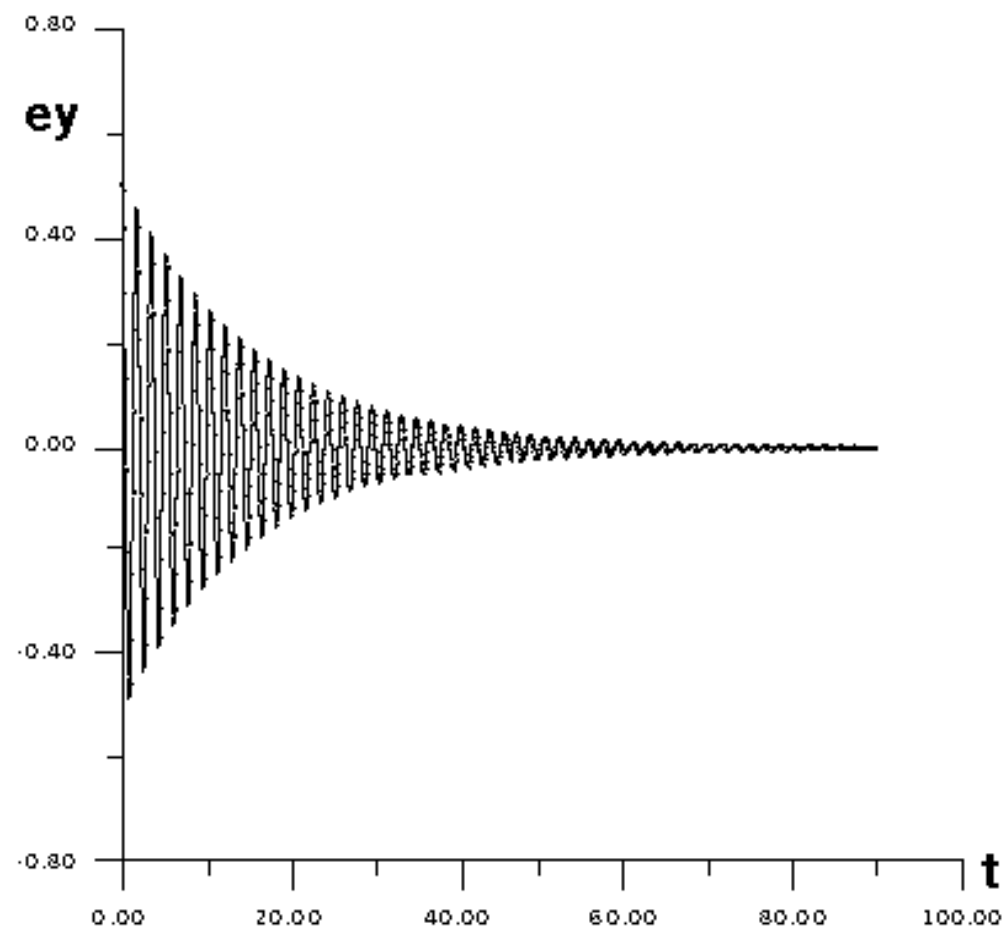

(b)

Fig. 11. Shows that the trajectory (a) $e_{x}$ of the error system converges to zero when $\alpha=9, \beta=100 / 7, a=-(8 / 7)$ and $b=-(5 / 7)$ where the controls $u_{3}=\tilde{k}_{3} e_{z}$ with $\dot{\tilde{k}}_{3}=4 e_{z}^{2}$ are activated at $t=15$. (b) $e_{y}$ of the error system converges to zero when $\alpha=9, \beta=100 / 7, a=-(8 / 7)$ and $b=-(5 / 7)$ where the controls $u_{3}=\tilde{k}_{3} e_{z}$ with $\dot{\tilde{k}}_{3}=3 e_{z}^{2}$ are activated at $t=6$. (c) $e_{z}$ of the error system converges to zero when $\alpha=9, \beta=100 / 7, a=-(8 / 7)$ and $b=-(5 / 7)$ where the controls $u_{3}=\tilde{k}_{3} e_{z}$ with $\dot{\tilde{k}}_{3}=3 e_{z}^{2}$ are activated at $t=4$. 


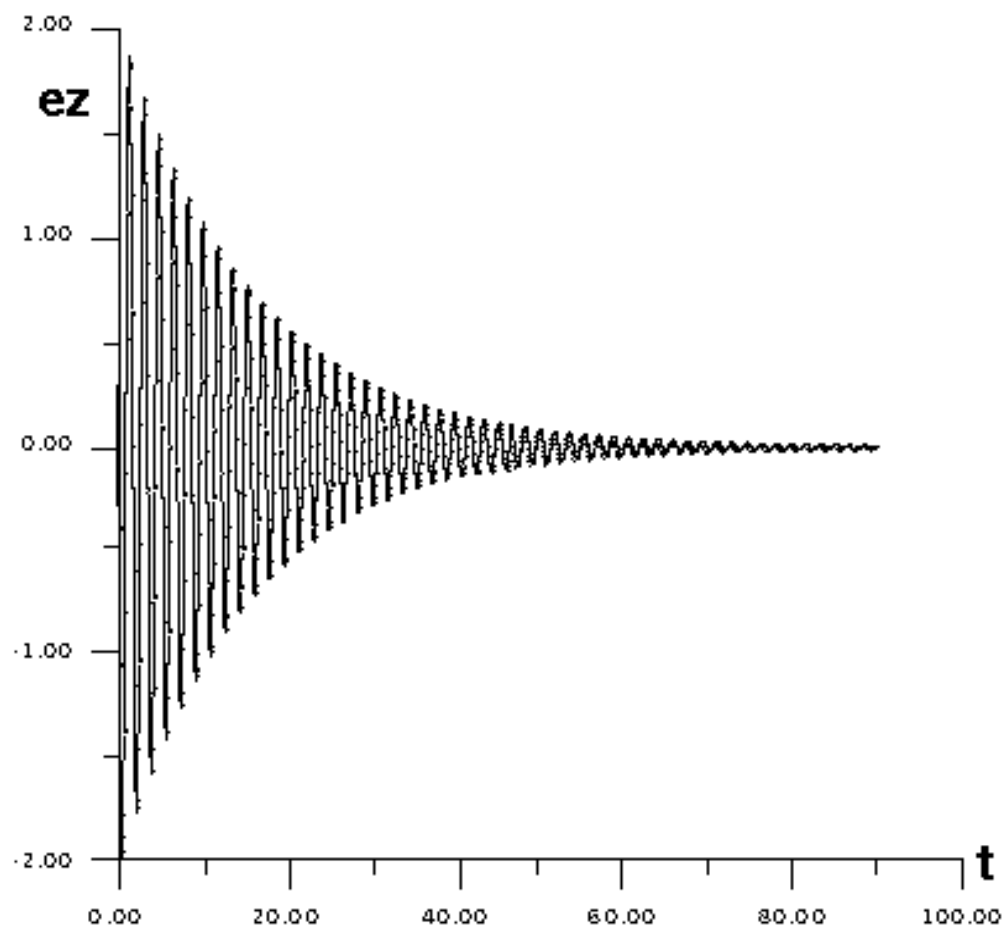

(c)

Fig. 11. (Continued)

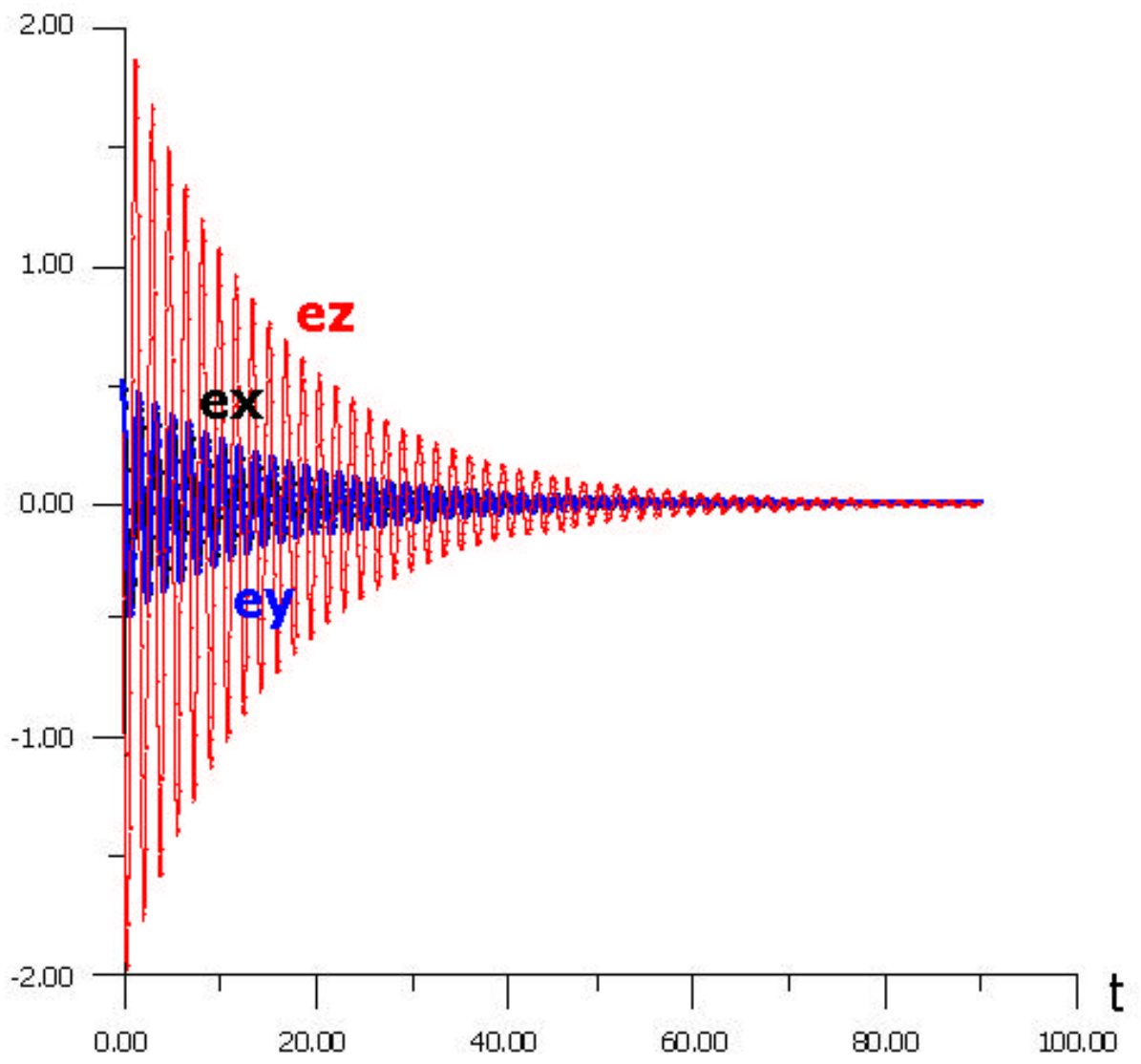

Fig. 12. Shows the state responses of adaptive synchronization when the adaptive control law is $u_{3}=\tilde{k}_{3} e_{z}$ with $\dot{\tilde{k}}_{3}=3 e_{z}^{2}$. 


\section{Conclusions}

In this paper we introduce adaptive synchronization problems for the Rössler and Chua's circuit systems, which are taken as examples of nonlinear dynamical systems of great importance in physics. The mathematical controllability conditions are derived from the Lyapunov direct method. As an example of a nonlinear dynamical system we have taken the Rössler and Chua's circuit systems which have great importance in physics. The first system (Rössler) after adding the adaptive control law $u_{1}$ and $u_{2}$ the trajectory $\left(e_{x}, e_{y}\right.$ and $\left.e_{z}\right)$ tended to zero after $t>10$, and after adding $u_{3}$ the trajectory tended to zero after $t>25$. The second system (Chua's circuit) after adding the adaptive control law $u_{1}$ the trajectory $\left(e_{x}, e_{y}\right.$ and $\left.e_{z}\right)$ tended to zero after $t>40$. After adding $u_{2}$ the trajectory tended to zero after $t>15$ and after adding $u_{3}$ the trajectory tended to zero after $t>4$.

\section{References}

Carroll, T. L. \& Pecora, L. M. [1991] "Synchronizing a chaotic systems," IEEE Trans. Circuits Syst. 38, 453-456.

Chen, G. \& Dong, X. [1993] "On feedback control of chaotic continuous-time systems," IEEE Trans. Circuits Syst. 40(9), 693-699.

Chen, G. [1997] "Control and synchronization of chaos," a Bibliography, Department of Elect. Eng., Univ. Houston, TX, available via ftp: uhoop.egr.uh.edu/pub/TeX/chaos.tex

Cuomo, K. M. \& Oppenheim, A. V. [1993] "Circuit implementation of synchronizing chaos with applications to communications," Phys. Rev. Lett. 71(1), 65-68.

Femat, R. \& Solis-Perales, G. [1999] "On the chaos synchronization phenomena," Phys. Lett. A1(262), $50-60$.

Hegazi, A. S., Agiza, H. N. \& El-Dessoky, M. M. [2001] "Synchronizatin and adaptive syncornization of nuclear spin generator system," Chaos Solit. Fract. 12(6), 1091-1099.

Hwang, C.-C., Chow, H.-Y. \& Wang, Y.-K. [1996] "A new feedback control of a modified Chua's circuit system," Physica D92, 95-100.

Hwang, C.-C., Hsieh, J.-Y. \& Lin, R.-S. [1997] "A linear continuous feedback control of Chua's circuit," Chaos Solit. Fract. 8(9), 1507-1515.

Kapitaniak, T. [1995] "Continuous control and synchronization in chaotic systems," Chaos Solit. Fract. 6(3), 237-244.

Kocarev, L. \& Parlitz, U. [1995] "General approach for chaotic synchronization with application to communication," Phys. Rev. Lett. 74(25), 5028-5031.

Kocarev, L. \& Parlitz, U. [1996] "Generalized syncronization, predictability, and equivalence of unidirectionally coupled dynamical systems," Phys. Rev. Lett. 76(11), $1816-1819$.

Liao, T.-L. \& Lin, S.-H. [1999] "Adaptive control and synchronization of Lorenz systems," J. Franklin Instit. 336, 925-937.

Matsumoto, T., Chua, L. O. \& Komuro, M. [1985] "The double scroll," IEEE Trans. Circuits Syst. 32, 798-818.

Parlitz, U., Junge, L. \& Kocarev, K. [1997] "Subharmonic entrainment of unstable period orbits and generalized synchronization," Phys. Rev. Lett. 79(17), 3158-3161.

Pecora, L. M. \& Carroll, T. L. [1990] "Synchronization in chaotic systems," Phys. Rev. Lett. 64(1), 821-824.

Pecora, L. M. \& Carroll, T. L. [1991] "Driving systems with chaotic signals," Phys. Rev. A44, p. 2374.

Pyragas, K. [1992] "Continuous control of chaos by selfcontrolling feedback," Phys. Lett. A170, 421-428.

Wu, C., Yang, T. \& Chua, L. O. [1996] "On adaptive synchronization and control of nonlinear dynamical systems," Int. J. Bifurcation and Chaos 6(3), $455-472$.

Xie, Q. \& Chen, G. [1996] "Synchronization stability analysis of the chaotic Rössler system," Int. J. Bifurcation and Chaos 6(11), 2153-2161.

Yang, X.-S. [1999] "Concepts of synchronization in dynamical systems," Phys. Lett. A260, 340-344.

Yang, X.-S. [2000] "A framework for synchroinzation theory," Chaos Solit. Fract. 11(9), 1365-1368.

Zeng, Y. \& Singh, S. N. [1996] "Adaptive control of chaos in Lorenz systems," Dyn. Contr. 7, 143-154. 